\title{
Universiteit
}

Leiden

The Netherlands

\section{Fluctuating pulled fronts: The origin and the effects of a finite particle} cutoff

Panja, D.; Saarloos, W. van

\section{Citation}

Panja, D., \& Saarloos, W. van. (2002). Fluctuating pulled fronts: The origin and the effects of a finite particle cutoff. Physical Review E, 66(3), 036206. doi:10.1103/PhysRevE.66.036206

Version: $\quad$ Not Applicable (or Unknown)

License: $\quad$ Leiden University Non-exclusive license

Downloaded from: https://hdl.handle.net/1887/66585

Note: To cite this publication please use the final published version (if applicable). 


\title{
Fluctuating pulled fronts: The origin and the effects of a finite particle cutoff
}

\author{
Debabrata Panja and Wim van Saarloos \\ Instituut-Lorentz, Universiteit Leiden, Postbus 9506, 2300 RA Leiden, The Netherlands \\ (Received 27 September 2001; revised manuscript received 13 May 2002; published 18 September 2002)
}

\begin{abstract}
Recently, it has been shown that when an equation that allows the so-called pulled fronts in the mean-field limit is modeled with a stochastic model with a finite number $N$ of particles per correlation volume, the convergence to the speed $v^{*}$ for $N \rightarrow \infty$ is extremely slow-going only as $\ln ^{-2} N$. Pulled fronts are fronts that propagate into an unstable state, and the asymptotic front speed is equal to the linear spreading speed $v^{*}$ of small linear perturbations about the unstable state. In this paper, we study the front propagation in a simple stochastic lattice model. A detailed analysis of the microscopic picture of the front dynamics shows that for the description of the far tip of the front, one has to abandon the idea of a uniformly translating front solution. The lattice and finite particle effects lead to a "stop-and-go" type dynamics at the far tip of the front, while the average front behind it "crosses over" to a uniformly translating solution. In this formulation, the effect of stochasticity on the asymptotic front speed is coded in the probability distribution of the times required for the advancement of the "foremost bin." We derive expressions of these probability distributions by matching the solution of the far tip with the uniformly translating solution behind. This matching includes various correlation effects in a mean-field type approximation. Our results for the probability distributions compare well to the results of stochastic numerical simulations. This approach also allows us to deal with much smaller values of $N$ than it is required to have the $\ln ^{-2} N$ asymptotics to be valid. Furthermore, we show that if one insists on using a uniformly translating solution for the entire front ignoring its breakdown at the far tip, then one can obtain a simple expression for the corrections to the front speed for finite values of $N$, in which various subdominant contributions have a clear physical interpretation.
\end{abstract}

DOI: 10.1103/PhysRevE.66.036206

\section{INTRODUCTION}

\section{A. Fronts and fluctuation effects}

In pattern forming systems, quite often, situations occur where patches of different bulk phases occur which are separated by fronts or interfaces. In such cases, the relevant dynamics is usually dominated by the dynamics of these fronts. When the interface separates two thermodynamically stable phases, as in crystal-melt interfacial growth problems, the width of the interfacial zone is usually of atomic dimensions. For such systems, one often has to resort to a moving boundary description in which the boundary conditions at the interface are determined phenomenologically or by microscopic considerations. A question that naturally arises for such interfaces is the influence of stochastic fluctuations on the motion and scaling properties of such interfaces.

At the other extreme is a class of fronts that arise in systems that form patterns, and in which the occurrence of fronts or transition zones is fundamentally related to their nonequilibrium nature, as they do not connect two thermodynamic equilibrium phases which are separated by a first order phase transition. In such cases-for example, chemical fronts [1], the temperature and density transition zones in thermal plumes [2], the domain walls separating domains of different orientation in in rotating Rayleigh-Bénard convection [3], or streamer fronts in discharges [4]-the fronts are relatively wide and therefore described by the same continuum equations that describe nonequilibrium bulk patterns. The lore in nonequilibrium pattern formation is that when the relevant length scales are large, (thermal) fluctuation effects are relatively small [5]. For this reason, the dynamics of many pattern forming systems can be understood in terms of the deterministic dynamics of the basic patterns and coherent
PACS number(s): 82.40.Bj, 05.10.Gg, 05.40.-a, 05.70.Ln

structures. For fronts, the first questions to study are therefore properties such as existence and speed of propagation of the front solutions of the deterministic equations, which in most cases are partial differential equations. In the last decades, the fundamental propagation mechanism of such deterministic fronts has become relatively well understood.

From the above perspective, it is may be less of a surprise that the detailed questions concerning the stochastic properties of inherently nonequilibrium fronts have been addressed, to some extent, only relatively recently [6-13], and that it has taken a while for researchers to become fully aware of the fact that the so-called pulled fronts [14-17] which propagate into an unstable state, do not fit into the common mold: they have anomalous sensitivity to particle effects [9-11], and have been argued to display uncommon scaling behavior [13,18-20].

Pulled fronts are fronts which propagate into an unstable state, and whose propagation dynamics is essentially that they are being "pulled along" by the growth and spreading of the small perturbations about the unstable state into which the front propagates-their asymptotic speed $v_{a s}$ is equal to the linear spreading speed $v^{*}$ of perturbations about the unstable state: $v_{a s}=v^{*}[14-17]$. This contrasts with the pushed fronts, for which $v_{a s}>v^{*}$, and whose dynamics is determined by the nonlinearities in the dynamical equations [1517]. The behavior of pushed fronts is essentially similar to fronts between two (meta)stable states.

The concept of a pulled front most naturally fits a formulation of the dynamical equations in terms of continuum variables, for by "small perturbations" we mean that the deviations of the field values from the values in the unstable state are small enough that nonlinear terms in the deviations can be neglected. From various directions, it has become clear in 
the last few years that such pulled fronts do show very unusual behavior and response to perturbations. First of all, Brunet and Derrida have shown that when the continuum field equations are used for a finite particle model so as to have a growth cutoff at the field value $1 / N$, where $N$ is the typical number of particles in the bulk phase behind the front, the deviation from the continuum value $v^{*}$ of the front speed is often large, and it vanishes only as $1 / \ln ^{2} N$ (with a known prefactor which they calculated) [9]. On the other hand, we recently found that with an infinitesimal growth cutoff and a similarly infinitesimal growth enhancement behind it, one can have a much higher front speed than $v^{*}$ [21]. Furthermore, the scaling properties of pulled fronts in stochastic field equations with a particular type of multiplicative noise have been found to be anomalous: in one dimension, they are predicted to exhibit subdiffusive wandering [18], but in higher dimensions their scaling behavior is given by the KPZ equation [22] in one dimension higher than one would naively expect $[19,20]$ (the question to what extent these results are applicable to lattice models, where the finite particle effects always make the fronts weakly pushed, is still a matter of debate [23,24]). Moreover, even without fluctuations, pulled fronts respond differently to coupling to other fields, e.g., they never reduce to standard moving boundary problems, even if they are thin [25].

All these effects have one origin in common, namely, the fact that the dynamics of pulled fronts, by its very nature, is not determined by the nonlinear front region itself, but by the region at the leading edge of the front, where deviations from the unstable state are small. To a large degree, this semiinfinite region alone determines the universal relaxation of the speed of a deterministic pulled front to its asymptotic value $[9,16,17]$, as well as the anomalous scaling behavior of stochastic fronts $[18-20,23,24]$ in continuum equations with multiplicative noise. As realized by Brunet and Derrida [9], the crucial importance of the region, where the deviations from the unstable state are small, also implies that if one builds a lattice model version of a front propagating into an unstable state, the front speed is surprisingly sensitive to the dynamics of the tip (the far end) of the front where only one or a few particles per lattice site are present. It is this effect which is the main subject of this paper.

\section{B. Open questions}

If we study fronts for a field describing the number density $\phi$ of particles, and normalize the field in such a way that its average value behind the front, where there are $N$ particles per unit of length, is 1 , then at the very far end of the leading edge, where the discrete particle nature of the actual model becomes most noticeable, the value of the normalized number density field is of order $1 / N$. Brunet and Derrida [9] therefore modeled the effect of the particle cutoff in their lattice model by studying a deterministic continuum front equation, in which the growth term was set to zero for values of $\phi$ less than $1 / N$. They showed that this led to a correction to the asymptotic front speed of the order of $1 / \ln ^{2} N$ with a prefactor, which is given in terms of the linear growth properties of the equation without a cutoff. Because of the loga- rithmic term, in the dominant order, it does not matter whether the actual cutoff should really be exactly $1 / N$ (corresponding to exactly one particle), or whether the growth is just suppressed at values of $\phi$ of order $1 / N$, since $1 / \ln ^{2}(c N)$ $\approx 1 / \mathrm{ln}^{2} N$ in dominant order. Simulations of two different lattice models by Brunet and Derrida [9] and by van Zon et al. [26] gave strong support for the essential correctness of this procedure for sufficiently large $N$, but showed that there can be significant deviations from the asymptotic result for large but not extremely large $N$. Moreover, for a different lattice model, Kessler et al. [10] did observe a correction to the average front speed of order $1 / \mathrm{ln}^{2} N$ but with a prefactor which they claimed was a factor of order two different from the prediction of Brunet and Derrida.

There are hence several questions that lead us to reconsider the finite particle effects on the average front speed of pulled stochastic fronts.

(i) Why is it that a simple cutoff of order $1 / N$ in a deterministic equation for a continuum (mean-field type) equation apparently leads to the proper asymptotic correction to the average speed of a stochastic front?

(ii) Can we get a more microscopic picture of the stochastic behavior at the far end of the front, where there are only a few particles per lattice site?

(iii) Can we go beyond the large $N$ asymptotic result of Brunet and Derrida, e.g., can we calculate the correction term for large but not extremely large values of $N$ or even for arbitrary $N$ ? After all, one might a priori expect correlation effects to be very important for fronts whose propagation speed is strongly affected by the region where there are only a few particles per site.

(iv) What is the role of correlation effects?

(v) To what extent do the specific details of the particular stochastic model play a role?

(vi) Can one resolve the discrepancy noted by Kessler et al. [10]?

\section{Summary of the main results}

In this paper, we address these questions and answer the majority of them for a specific model for which Breuer et al. [6] already studied the asymptotic speeds of stochastic fronts numerically some years ago. The model consists of particles making diffusive hops on a one-dimensional lattice, and being subject to growth and death on each lattice site. It is very close to the one also studied by Kessler et al. [10], the only difference being that their model includes a correlation term, which is small and irrelevant for large $N$. The absence of such correlations makes the model studied by Breuer et al. easier to analyze. Moreover, an examination of the numerical results therein shows that the deviation of the asymptotic front speed from its pulled front value indeed behaves as $1 / \ln ^{2} N$ (although it was not realized in Ref. [6]), with a prefactor that, over the range of $N$ values studied, is different from the one predicted later by Brunet and Derrida [9], but not as much different as Kessler et al. claimed it to be for their own model [10]. For each stochastic realization of a front, which moves into a region where no particles are present, one can always identify a foremost occupied lattice 
site or "the foremost bin." In the region near this one, fluctuations are large and the discreteness of the lattice and of the particle number occupation is extremely important: the standard description, which assumes that the average particle density is uniformly translating, breaks down in this region. Moreover, since the particle occupation numbers are small in the tip, essentially all known methods fail, based as they are on large- $N$ expansions.

With a finite particle cutoff, fronts are never really pulled, but instead are weakly pushed [27]. Even for the simplest case of a pushed front in a second order nonlinear partial differential equation, in general, the speed cannot be calculated explicitly. It should therefore come as no surprise that with the various additional complications described above, we do not have a full first principles theory that gives the front speed for finite values of $N$ for the model we study. However, in this paper we do obtain a number of important results for the behavior in the far tip of the front as well as for the effect of the region behind the tip on the finite- $N$ corrections. These results can be tested independently and our numerical simulations largely support the picture that emerges from this approach. In terms of short answers to the questions raised above in Sec. I B, we find that

(i) For extremely large $N$, the asymptotic results of Brunet and Derrida based on a simple cutoff of order $1 / N$ in a $d e-$ terministic equation for a continuum (mean-field type) equation become essentially correct because all the essential changes are all limited to a few bins behind the foremost one, where the particle numbers are finite and small; together with the fact that $1 / \ln ^{2}(c N) \approx 1 / \ln ^{2}(N)$ to dominant order, this ensures the correctness of the asymptotic expression for $N$ $\rightarrow \infty$.

(ii) Yes, one can get a more microscopic picture of what happens near the foremost bin of the front; we develop mean-field type expressions for the probability distribution that describes the "stop-and-go" type behavior there (Sec. IV), and show that the results compare well with numerical simulation results, Sec. V.

(iii) A first-principles theory for the stochastic front speed for arbitrary $N$ seems virtually impossible, except possibly in some special limits, as in principle, it will involve matching the approximately uniformly translating average profile behind the tip of the front to the nonuniformly translating profile near the foremost bin, where standard methods do not seem to apply.

(iv) Correlation effects are very important near the tip; we identify two of them and model one: rapid successive forward hops of the foremost particle, Sec. IV C 1, and jumping back of the foremost particle, Sec. IV C 2.

(v) The details of the particular stochastic model play a role for the corrections in the asymptotic front speed through the global average front profile (quantified by $A$ of Secs. III and IV) and through the effective profile near the tip, but their effects are truly minute. We demonstrate this by means of a mean-field theory that tries to extend the uniformly translating front solution all the way to the far tip of the front (described in Sec. III C). In this theory, there is a quantity $a$ associated with the effective profile at the tip, and we show that these two quantities, $A$ and $a$, provide only subdominant corrections to the asymptotic large $N$ result.

(vi) The model considered by Kessler et al. [10] is slightly different from the one considered by Breuer et al., in the sense that number of particles of each species is finite. However, a priori, one expects that this difference in the two models would not affect the speed corrections for large $N$. Our own simulations confirm this, and show no sign of a discrepancy between the asymptotic large- $N$ speed corrections obtained from the two models (Sec. VI).

We finally note that in this paper, we will focus on the case where the growth and hopping terms for a few particles are the same as those for a small but finite density of particles. In such cases, the front speed converges for $N \rightarrow \infty$ to the pulled front speed $v^{*}$ of the corresponding mean-field equation. As we will discuss elsewhere [21], with only slight modifications of the stochastic rules for few particles, one can also arrive at situations where the limits do not commute, i.e., where the stochastic front speed converges to a speed larger then $v^{*}$ as $N \rightarrow \infty$, even though the stochastic model would converge to the mean-field equation with pulled fronts in this limit.

\section{Complications associated with discreteness of the lattice and particle numbers}

The challenge of understanding the propagation of any one of these fronts lies in the fact that as a consequence of the discrete nature of the particle events and of the particle number realizations, the natural description of the far tip is not in terms of a uniformly translating solution for the average number of particles in the bins (we call each lattice site a "bin"), but is in terms of discrete notions such as the foremost bin, individual jumps, etc. An additional complication is that in the presence of fluctuations, the front position exhibits diffusionlike wandering behavior, which have to be taken out in order to study the intrinsic stochastic front dynamics, just like capillary waves beset analyzing the intrinsic structure of a fluid interface (Sec. III B). The implication of all this is that (i) in the presence of an underlying lattice, instead of being uniformly translating, the position of the foremost occupied bin advances in a discrete manner, and (ii) due to the discrete nature of the constituent particles, the position of the foremost bin advances probabilistically, as its movement is controlled by diffusion.

Based on these ingredients and observations, the central theme in this paper revolves around a picture of the tip of the front that is totally different from the conventional picture of a pulled front. We present the picture here in terms of its simplified essence, as it is helpful for the reader to bear it in mind throughout this paper: we call the foremost occupied lattice site at the far end of the tip of the front "the foremost bin." Therefore, the very definition of the foremost bin on a lattice site means that it is occupied by at least one particle and that all the lattice sites on the right of it are empty. Naturally, an empty lattice site (all the lattice sites on the right of which are also empty) attains the status of the foremost bin as soon as one particle hops into it from the left. In reference to the lattice, the position of the foremost bin remains fixed at this site for some time, i.e., after its creation, 
a foremost bin remains the foremost bin for some time. During this time, however, the number of particles in and behind the foremost bin continues to grow. As the number of particles grows in the foremost bin, the chance of one of them making a diffusive hop on to the right also increases. At some instant, a particle from the foremost bin hops over to the right: as a result of this hop, the position of the foremost bin advances by one unit on the lattice, or, viewed from another angle, a new foremost bin is created which is one lattice distance away on the right of the previous one. Microscopically, the selection process for the length of the time span between two consecutive foremost bin creations is stochastic, and the inverse of the long time average of this time span defines the front speed. Simultaneously, the amount of growth of particle numbers in and behind the foremost bin itself depends on the time span between two consecutive foremost bin creations (the longer the time span, the longer the amount of growth). As a consequence, on average, the selection mechanism for the length of the time span between two consecutive foremost bin creations, which determines the asymptotic front speed, is nonlinear.

This inherent nonlinearity makes the prediction of the asymptotic front speed difficult. One might recall the difficulties associated with the prediction of pushed fronts due to nonlinear terms in this context, although the nature of the nonlinearities in these two cases is completely different. In the case of pushed fronts, the asymptotic front speed is determined by the mean-field dynamics of the fronts, and the nonlinearties originate from the nonlinear growth terms in the partial differential equations that describe the mean-field dynamics (as we discuss in Sec. III B, if one does not take out the wandering of the front positions, then the nonlinear growth terms actually do affect the stochastic front dynamics in a subtle way too). On the other hand, for fronts consisting of discrete particles on a discrete lattice, the corresponding mean-field growth terms are linear, but since the asymptotic front speed is determined from the probability distribution of the time span between two consecutive foremost bin creations, on average, it is the relation between this probability distribution and the effect of the linear growth terms that the nonlinearities stem from.

Our approach is to develop a separate probabilistic theory for the hops to create the new foremost bins, and then to show that by matching the description of the behavior in this region to the more standard one (of growth and roughly speaking, uniform translation) behind it, one obtains a consistent and more complete description of the stochastic and discreteness effects on the front propagation. In the simplest approximation, the theory provides a very good fit to the data, but our approach can be systematically improved by incorporating the effect of fluctuations as well. Besides providing insight into how a stochastic front propagates at the far tip of the leading edge, our analysis naturally leads to a more complete description that allows one to interpret (though not predict) the finite $N$ corrections to the front speed for much smaller values of $N$ than that are necessary to see the asymptotic result of Brunet and Derrida [9]. As one might expect, for values of $N$ where deviations from this asymptotic result are important, model-specific effects do play a role.

For the major part of our analysis, we focus on the most relevant and illuminating case in which the diffusion and growth rates of the model are both of the same order. This regime is the most illustrative as it displays all the aspects of finite particle and lattice effects most clearly. We also investigate the case when the diffusion rate is much smaller than the growth rate to illustrate the correlation effects. For all of these cases, the matching between the behavior of the tip of the front and the standard description of a uniformly translating solution behind it is a complicated process, for the lack of a proper small parameter that allows one to do perturbation theory.

The paper is organized in the following manner, in Sec. II, we describe our model (which is the same as in Ref. [6]) and define the dynamics of the front. The crux of the paper is presented in Sec. IV, where we present a detailed analysis of the microscopic picture of the front dynamics and show that for the description of the far tip of the front, one has to abandon the idea of a uniformly translating front solution. The lattice and finite particle effects lead to a "stop-and-go" type dynamics at the far tip of the front, while the average front behind it "crosses over" to a uniformly translating solution. In this formulation, the effect of stochasticity on the asymptotic front speed is coded in the probability distribution of the times required for the advancement of the foremost bin. We derive expressions of these probability distributions by matching the solution of the far tip with the uniformly translating solution behind. This matching includes various correlation effects in a mean-field type approximation. In Sec. V, we compare our theoretical predictions of Sec. IV with the stochastic simulation results. In addition to that, in Sec. III, we argue that the corresponding front solution is a case of a weakly pushed front and analyze an effective mean-field solution that extends all the way to the foremost bin (thereby ignoring its breakdown near the foremost bin). This allows us to rederive the asymptotic velocity expression of Brunet and Derrida [9] and obtain the further subdominant finite- $N$ corrections to it. In Sec. VI, we carry out the full stochastic simulation for the model considered by Kessler et al., and finally, we conclude the paper with a discussion and outlook in Sec. VII.

\section{THE REACTION-DIFFUSION PROCESS $X+Y \rightleftharpoons 2 X$ ON A LATTICE}

We consider the following reaction-diffusion process $X$ $+Y \rightleftharpoons 2 X$ on a lattice in the following formulation: at each lattice position, there exists a bin. We label the bins by their serial indices $k, k=1,2,3, \ldots, M$, placed from left to right. Each bin has an infinite supply of $Y$ particles. An $X$ particle in the $k$ th bin can undergo three basic processes: (i) diffusion to the $(k-1)$ th or the $(k+1)$ th bin with a rate of diffusion $\gamma$. If an $X$ particle in bin 1 jumps towards the left, or an $X$ particle in the $M$ th bin jumps to the right, then they are immediately replaced, (ii) forward reaction to produce an extra $X$ particle having annihilated a $Y$ particle $(X+Y \rightarrow 2 X)$, with a rate $\gamma_{g}$, 
and (iii) if there are at least two $X$ particles present in the $k$ th bin, then any two of the $X$ particles can react with each other and annihilate one $X$ particle to produce a $Y$ particle $(2 X$ $\rightarrow X+Y$ ), with a reaction rate $\gamma_{d}$. The state of the system at time $t$ is given by the numbers of $X$ particles in the bins, denoted as $\left\{N_{1}, N_{2}, \ldots, N_{M} ; t\right\}$.

In the context of front propagation, the above model was first studied by Breuer et al. [6]. Up to Sec. V of this paper, we will confine ourselves to this model only. In Sec. VI, we will consider a slightly modified version of this model, numerically studied by Kessler and coauthors [10], in which the number of $Y$ particles in any bin is finite, and the $Y$ particles can diffuse from any bin to its nearest neighbor bins with the same diffusion rate $\gamma$.

\section{A. The master equation}

The discrete, microscopic description of the above reaction-diffusion process inherently introduces fluctuations in the number of $X$ particles present in any particular bin. This necessitates a suitable multivariate probabilistic description of the system. Let us denote the probability of a certain configuration $\left\{N_{1}, N_{2}, \ldots, N_{M} ; t\right\}$ at time $t$ by $P\left(N_{1}, N_{2}, \ldots, N_{M} ; t\right)$. The dynamics of $P\left(N_{1}, N_{2}, \ldots, N_{M} ; t\right)$ is given by the following master equation:

$$
\begin{aligned}
\frac{\partial}{\partial t} P\left(N_{1}, N_{2}, \ldots, N_{M} ; t\right)= & \sum_{k}\left\{\gamma \left[\left(N_{k+1}+1\right) P\left(N_{1}, N_{2}, \ldots, N_{k}-1, N_{k+1}+1, \ldots, N_{M} ; t\right)\right.\right. \\
& +\left(N_{k-1}+1\right) P\left(N_{1}, N_{2}, \ldots, N_{k-1}+1, N_{k}-1, \ldots, N_{M} ; t\right) \\
& \left.-2 N_{k} P\left(N_{1}, N_{2}, \ldots, N_{k-1}, N_{k}, N_{k+1}, \ldots, N_{M} ; t\right)\right] \\
& +\gamma_{g}\left[\left(N_{k}-1\right) P\left(N_{1}, N_{2}, \ldots, N_{k-1}, N_{k}-1, N_{k+1}, \ldots, N_{M} ; t\right)\right. \\
& \left.-N_{k} P\left(N_{1}, N_{2}, \ldots, N_{k-1}, N_{k}, N_{k+1}, \ldots, N_{M} ; t\right)\right] \\
& +\frac{\gamma_{d}}{2}\left[N_{k}\left(N_{k}+1\right) P\left(N_{1}, N_{2}, \ldots, N_{k-1}, N_{k}+1, N_{k+1}, \ldots, N_{M} ; t\right)\right. \\
& \left.\left.-N_{k}\left(N_{k}-1\right) P\left(N_{1}, N_{2}, \ldots, N_{k-1}, N_{k}, N_{k+1}, \ldots, N_{M} ; t\right)\right]\right\} .
\end{aligned}
$$

The above equation is actually not quite accurate at the 1 st and $M$ th boundary bins, but we refrain from writing out the correction terms explicitly, as they are not needed in the analysis below.

\section{B. The macroscopic density field and the Fisher-Kolmogorov equation}

If the forward reaction rate, $\gamma_{g}$, is much larger than the annihilation rate $\gamma_{d}$, an initial conglomeration of $X$ particles will start to grow in size as well as in numbers. To study this growth phenomena, we define $\left\langle N_{k}(t)\right\rangle$, the average number of $X$ particles in the $k$ th bin at time $t$, as

$$
\left\langle N_{k}(t)\right\rangle=\sum_{\left\{N_{k^{\prime}}\right\}_{k^{\prime}=1 \ldots N}} N_{k} P\left(N_{1}, N_{2}, \ldots, N_{M} ; t\right) .
$$

Using Eq. (2.1), it is easy to obtain the time dynamics of $\left\langle N_{k}(t)\right\rangle$, given by

$$
\begin{aligned}
\frac{\partial}{\partial t}\left\langle N_{k}(t)\right\rangle= & \gamma\left[\left\langle N_{k+1}(t)\right\rangle+\left\langle N_{k-1}(t)\right\rangle-2\left\langle N_{k}(t)\right\rangle\right] \\
& +\gamma_{g}\left\langle N_{k}(t)\right\rangle-\frac{\gamma_{d}}{2}\left[\left\langle N_{k}^{2}(t)\right\rangle-\left\langle N_{k}(t)\right\rangle\right],
\end{aligned}
$$

with

$$
\left\langle N_{k}^{2}(t)\right\rangle=\sum_{\left\{N_{k^{\prime}}\right\} k^{\prime}=1 \cdots N} N_{k}^{2} P\left(N_{1}, N_{2}, \ldots, N_{M} ; t\right) .
$$

For the sake of simplicity, we define $\tilde{\gamma}=\gamma / \gamma_{g}, t^{\prime}=\gamma_{g} t$, and $N=2 \gamma_{g} / \gamma_{d}$, and reduce the number of parameters in Eq. (2.3), to have [6]

$$
\begin{aligned}
\frac{\partial}{\partial t^{\prime}}\left\langle N_{k}\left(t^{\prime}\right)\right\rangle= & \tilde{\gamma}\left[\left\langle N_{k+1}\left(t^{\prime}\right)\right\rangle+\left\langle N_{k-1}\left(t^{\prime}\right)\right\rangle-2\left\langle N_{k}\left(t^{\prime}\right)\right\rangle\right] \\
& +\left\langle N_{k}\left(t^{\prime}\right)\right\rangle-\frac{1}{N}\left[\left\langle N_{k}^{2}\left(t^{\prime}\right)\right\rangle-\left\langle N_{k}\left(t^{\prime}\right)\right\rangle\right] .
\end{aligned}
$$

Following the procedure in Ref. [6], if one replaces the $(1 / N)\left[\left\langle N_{k}^{2}(t)\right\rangle-\left\langle N_{k}(t)\right\rangle\right]$ term in Eq. (2.5) by $(1 / N)\left\langle N_{k}(t)\right\rangle^{2}$ and further defines a mean "concentration field" on the $k$ th bin by introducing the variable $\phi_{k}$ $=\left\langle N_{k}\right\rangle / N$, then from Eq. (2.5), one arrives at the following difference-differential version of the Fisher-Kolmogorov equation for the reaction-diffusion process $X+Y \rightleftharpoons 2 X$ on a lattice, given by [6] 


$$
\frac{\partial}{\partial t} \phi_{k}(t)=\tilde{\gamma}\left[\phi_{k+1}(t)+\phi_{k-1}(t)-2 \phi_{k}(t)\right]+\phi_{k}(t)-\phi_{k}^{2}(t) .
$$

The original Fisher-Kolmogorov or FKPP equation $[28,29]$ is a partial differential equation in continuous space and time. Notice that in these variables, the properties of the propagating front depend only on two parameters, $N$ and $\tilde{\gamma}$.

\section{MEAN-FIELD APPROXIMATIONS TO THE PROPAGATING FRONT SOLUTION}

As mentioned earlier, in this section we do not consider the proper stop-and-go type dynamics of the tip; instead, as a continuation of mean-field equation (2.6) above, we describe the entire front by the uniformly translating profile. We then make a number of general observations concerning the uniformly translating front solutions in mean-field type equations for the average profile, from the perspective of the questions raised in the Introduction. A central result of the discussion will be an expression for the finite- $N$ value of the velocity, which allows us to interpret deviations from the asymptotic results of Ref. [9] in terms of physical properties of stochastic fronts.

\section{A. Front propagation in the dynamical equation for $\phi_{k}(t)$}

From the point of view of average number of $X$ particles in the bins, Eq. (2.5) has two stationary states. One of them, for which $\left\langle N_{k}\right\rangle=N, \forall k$, is stable. The other, for which $\left\langle N_{k}\right\rangle=0, \forall k$, is unstable. This means that any perturbation around the unstable state grows in time until it saturates at the stable state value. In particular, if in a certain configuration of the system, the stable and the unstable regions coexist, i.e., $\left\langle N_{k}\right\rangle=N, \forall k<k_{0}$ and $\left\langle N_{k}\right\rangle=0, \forall k>k_{1}$, with $k_{1}$ $>k_{0}$, then the stable region invades the unstable region and propagates into it. In other words, in due course of time, the boundary between these two regions, having a finite width, moves further and further inside the unstable region. For a wide range of initial conditions, the speed, with which this boundary moves into the unstable region, approaches a fixed asymptotic value, $v_{a s}$. Simultaneously, the shape of this boundary between the two regions, determined by the average number of $X$ particles, $\left\langle N_{k}\right\rangle$, plotted against the corresponding bin indices $k$, also approaches an asymptotic shape. This asymptotic shape, therefore, becomes a function of ( $k$ $\left.-v_{a s} t\right)$ for long times, and this well-known phenomenon is known as the front propagation. In the present context, Eqs. (2.5)-(2.6) provide us with an example of front propagation into unstable states. We will follow the usual convention that the front propagates to the right in the direction of increasing bin numbers.

In the mean-field approximation (2.6), the average particle density field $\phi_{k}(t)$ obeys a difference-differential equation. The asymptotic speed selection mechanism for propagating fronts into unstable states has been a well-understood phenomenon for a number of years, and it has been realized by various authors $[9-11,17]$ that the calculation of the asymptotic front speed on a lattice for the type of Eqs. (2.5)-
(2.6) proceeds along similar lines as it does for partial differential equations. It is well-known that for Eqs. (2.5)-(2.6), the selection mechanism for $v_{a s}$ depends entirely on the region, where the nonlinear saturation terms $\left(\left[\left\langle N_{k}^{2}(t)\right\rangle\right.\right.$ $\left.-\left\langle N_{k}(t)\right\rangle\right] / N$ or $\left.\phi_{k}^{2}(t)\right)$ are much smaller in magnitude than the corresponding linear growth terms $\left[\left\langle N_{k}(t)\right\rangle\right.$ or $\left.\phi_{k}(t)\right]$, i.e., the leading edge of the front, where the value of $\phi_{k}(t)$ is very small, such that $\phi_{k}^{2}(t) \ll \phi_{k}(t)$. In this region, the nonlinear terms can be neglected, and after having used $\phi_{k}(t)$ $\equiv \phi\left(k-v_{a s} t\right) \equiv \phi(\xi)$, where $\xi=k-v_{a s} t$ is the comoving coordinate, Eq. (2.6) reduces to a linear differencedifferential equation, given by

$$
-v_{a s} \frac{\partial}{\partial \xi} \phi(\xi)=\tilde{\gamma}[\phi(\xi+1)+\phi(\xi-1)-2 \phi(\xi)]+\phi(\xi) .
$$

If one neglects the fact that the microscopic $X$ particles are discrete and assumes that $\phi(\xi)$ goes to zero continuously for $\xi \rightarrow \infty$, then a natural candidate for the solution of $\phi(\xi)$ in the linear difference-differential equation, Eq. (3.1) above, is $\phi(\xi) \equiv A \exp [-z \xi]$, where $z$ is a real and positive quantity. With this solution of $\phi(\xi)$ in the so-called leading edge of the front, one arrives at the dispersion relation

$$
v_{a s} \equiv v_{a s}(z)=\frac{2 \tilde{\gamma}[\cosh (z)-1]+1}{z} .
$$

Like the other examples of fronts propagating into unstable states, Eq. (3.2) allows an uncountably infinite number of asymptotic velocities depending on the selected value of the continuous parameter $z$. However, for a steep enough initial condition that decays faster than $\exp (-z \xi)$ in $\xi$ for any $z>z_{0}$ determined below (hence, a unit step function obeys this condition), the observed asymptotic speed equals the socalled linear spreading speed $v^{*}$, given by $v^{*} \equiv v^{*}\left(z_{0}\right)$, where $z_{0}$ is the value of $z$, for which the dispersion relation $v_{a s}(z)$ vs $z$ has a minimum.

The fact that $v^{*}$ defined in this way is nothing but the linear spreading speed, i.e., the spreading speed of small perturbations whose dynamics is given by the linearized equation (3.2), follows from a saddle point analysis of the asymptotic behavior of the Green's function for the linear equation (3.2), see, e.g., Ref. [17]. The name pulled fronts stems from the fact that this linear spreading almost literally "pulls" the nonlinear front with it, the nonlinear terms just giving rise to saturation behind the front.

\section{B. The deceptive subtlety of the mean-field approximation}

As we discussed above, in the pulled front regime, the front speed of a given problem is determined completely by the linear term in the dynamical equation. In going from the exact equation (2.5) to the mean-field approximation (2.6), we appear, at first sight, to have ignored only a term linear in $\phi_{k}$ of order $1 / N$ [the second term between square brackets in Eq. (2.5)]. Hence, naively one might expect the front speed to converge as $1 / N$ to the asymptotic value $v^{*}\left(z_{0}\right)$. We already know from the work of Brunet and Derrida [9], how- 
ever, that the convergence is much slower, namely, as $1 / \ln ^{2} N$. How can the two results be reconciled?

The resolution of the paradox lies in the fact that in the mean-field approximation we completely ignore the diffusive wandering of fronts. If we follow the evolution of an ensemble of fronts, their positions [defined, e.g., by Eq. (4.1) below] will fluctuate: the root mean square wandering of the fronts grows as $\sqrt{t}$ as for any one-dimensional random walker $[6,12]$. This means that in reality the ensemble average $\left\langle N_{k}(t)\right\rangle$ does not acquire a fixed shape in the frame moving with the average speed. Instead, the average profile $\left\langle N_{k}(t)\right\rangle$ continues to broaden in time, although the front shapes for the individual realizations reach an asymptotic shape (see Fig. 5 of Ref. [6] for an illustration). This has a severe consequence: we cannot simply assume that the $\left\langle N_{k}^{2}(t)\right\rangle$ term is small in the leading edge of the profile where $\left\langle N_{k}(t)\right\rangle$ is small, and replace it by $\left\langle N_{k}(t)\right\rangle^{2}$-few members of the ensemble, which are relatively further ahead, do give significant contributions through this term in regions where $\left\langle N_{k}(t)\right\rangle$ is small. Thus, while Eq. (2.5) is exact and contains the fluctuation effects due to the root mean square wandering of the front, the mean-field approximation (2.6) throws out such effects completely.

If, on the other hand, we look at the shape of a particular front realization in the appropriate position, so that the front wandering is taken out, the mean-field equation does yield a reasonably good description of this (conditionally averaged) front profile in the range where the particle occupation numbers are large and (hence) where fluctuation effects are small. Additional information is needed, however, to calculate the front speed.

In passing, we note that the situation is somewhat similar to the theory of fluid interfaces: capillary wave fluctuations wash out the average interface profile completely, but on scales of the order of the capillary length, the mean-field theory for the so-called intrinsic interface profile works quite well.

\section{The front speed correction for large $N$}

The above observations already allow us to arrive at and extend the results of Brunet and Derrida [9] from a slightly different angle than in their original work as follows. First of all, from the discussion above, we notice that even though a mean-field approximation (2.6) does not work for the ensemble-averaged front profile, but for a given stochastic front realization, the mean-field theory does apply to a good approximation in the bins, where the number of $X$ particles are relatively large. These are essentially the bins that are sufficiently behind the foremost bin, the rightmost bin in the given stochastic realization, on the right of which all bins are completely empty. Nevertheless, as mentioned in the beginning paragraph of this section, we assume that the uniformly translating front solution of Eq. (2.6) holds for the description of the front profile all the way up to the foremost bin for a given realization. Second, the actual front solution of Eq. (2.6) is a case of a weakly pushed front as opposed to being a truly pulled front $[27,30]$. This can be understood in the following manner: notice that in any bin the forward reaction
$X+Y \rightarrow 2 X$ does not proceed unless there is at least one $X$ particle in that bin to start with. As for any given realization of the stochastic front, the front propagation on a lattice is tantamount to the discrete forward movement of the foremost bin by units of 1 (which can happen only through the diffusion of an $X$ particle from the foremost bin towards the right), in the uniformly translating front solution of Eq. (2.6), the dynamics of the tip of the front is diffusion dominated. This makes any given realization of the front weakly pushed as opposed to being truly pulled, and moreover, the asymptotic speed $v_{N}$ is expected to be $<v^{*}$ for a finite $N$. This indicates that if we want to build all these in the same frame as in the velocity selection mechanism for a pulled front, one has to allow complex values of the parameter $z$ [see Eq. (3.2) and the discussion thereabove]. Furthermore, the existence of a foremost bin requires that the front profile must have a zero a bin ahead of the foremost bin. Having combined all these together, and without any loss of generality, we now require that the front profile in the linear region of Eq. (2.6) is given by $[9-11,16,17]$ for $\phi(\xi)$ for $v_{N}<v^{*}$,

$$
\phi(\xi)=A \sin \left[z_{i} \xi+\beta\right] \exp \left(-z_{r} \xi\right),
$$

such that $\phi(\xi)$ has a node at the coordinate of the bin just ahead of the foremost bin. In Appendix A, we show how Eq. (3.3) can be used to determine the complex decay rate $z$ in terms of $N$ and other parameters, and from that we obtain the deviation of the front speed $v_{N}$ from $v^{*}$. The front speed $v_{N}$ is given by

$$
\begin{gathered}
v_{N}=v^{*}-\left.\frac{d^{2} v_{a s}}{d z^{2}}\right|_{z_{0}} z_{i}^{2}+O\left(z_{i}^{4}\right) \approx v^{*}-\left.\frac{d^{2} v_{a s}}{d z^{2}}\right|_{z_{0}} \\
\times \frac{\pi^{2} z_{0}^{2}}{\left[\ln N+z_{0}+\ln \frac{A}{a}+\ln \left\{\sin \frac{\pi z_{0}}{\ln N+1}\right\}\right]^{2}},
\end{gathered}
$$

where, according to Eq. (3.2),

$$
\left.\frac{d^{2} v_{a s}}{d z^{2}}\right|_{z_{0}}=\frac{\tilde{\gamma} \cosh z_{0}}{z_{0}} .
$$

In the limit of large $N$, the above result (3.4) reduces to

$$
v_{N} \approx v^{*}-\left.\frac{d^{2} v_{a s}}{d z^{2}}\right|_{z_{0}} \frac{\pi^{2} z_{0}^{2}}{\ln ^{2} N},
$$

which is nothing but the asymptotic expression for the velocity correction derived by Brunet and Derrida [9]. Their approach is based on the partial differential equation analog of the mean-field dynamical equation (2.6), in that they introduced an artificial cutoff for the growth term for values of $\phi(\xi)<\varepsilon$, where $\varepsilon \approx 1 / N$, to mimic the dominant role played by diffusion at the tip of the front as opposed to the growth term. 


\section{Implications and discussion}

The above expressions for the speed corrections are already quite instructive. First of all, as we pointed out, for the speed difference $v^{*}-v_{N}$, Eq. (3.5) reduces to the expression of Eq. (3.6) of Brunet and Derrida [9] at the dominant order in the limit of very large $N$. To this order, the speed change is given explicitly in terms of $N$. The more general expression, Eq. (3.5), however, contains the factors $A$ and $a$; these affect the subdominant behavior, i.e., the corrections to the asymptotic large $N$ expression. For realistic values of $N$, the corrections to the asymptotic behavior can be quite significant [9]. As we shall show in Sec. V B, A depends on the global behavior of the average front solution, including the behavior in the region where nonlinearities are important. This makes its value vary from model to model and it is at this place where the specific details of the model affect the speed difference $v^{*}-v_{N}$. On the other hand, $a$ is only a parameter that originates through the extrapolation of the mean-field profile (3.3) to the foremost bin region. We will show in the following section that the quantity $a$ is a fictitious quantity, as the average front profile deviates significantly from the one in Eq. (3.3) near the foremost bin: as we shall see, unlike the mean-field solution, it is not even uniformly translating. This is the reason that an explicit general prediction for the front speed beyond the asymptotic result obtained by Brunet and Derrida [9] is hard, if not impossible, to come by.

In passing, we note the following. It is well known from the analysis of uniformly translating front solutions of the Fisher-Kolmogorov partial differential equation that front solutions with $v<v^{*}$ are asymptotically given by an expression like Eq. (3.3), and that these front solutions with nodes are unstable. This does not mean, however, that the above (crude) analysis is based on an unstable solution (3.3) and therefore inconsistent. The point is that the expression (3.3) is only an intermediate asymptotic solution, valid over some finite range of bins; just as in the analysis of the slow time relaxation of pulled fronts in partial differential equations [17], where such solutions also play a role as intermediate asymptotics, but they do not make the full solution unstable.

\section{THE PROBABILISTIC DYNAMICS OF THE TIP: BREAKDOWN OF THE DEFINITION OF THE COMOVING COORDINATE $\xi$}

We now turn to the analysis of the stochastic dynamics near the foremost bin, which is the region which determines most of the front dynamics. In the light of the discussion of Sec. III B, from here onwards, we confine ourselves to the study of one single front realization.

Let us assume that as the front moves in time from the left to the right, at some time $t=t_{0}$, the bin $k_{0}$ is deep inside the saturation phase of the front. At time $t \geqslant t_{0}$, the total number of particles on the right of the $k_{0}$ th bin is given by

$$
N_{t o t}(t)=\sum_{k>k_{0}} N_{k}(t)
$$
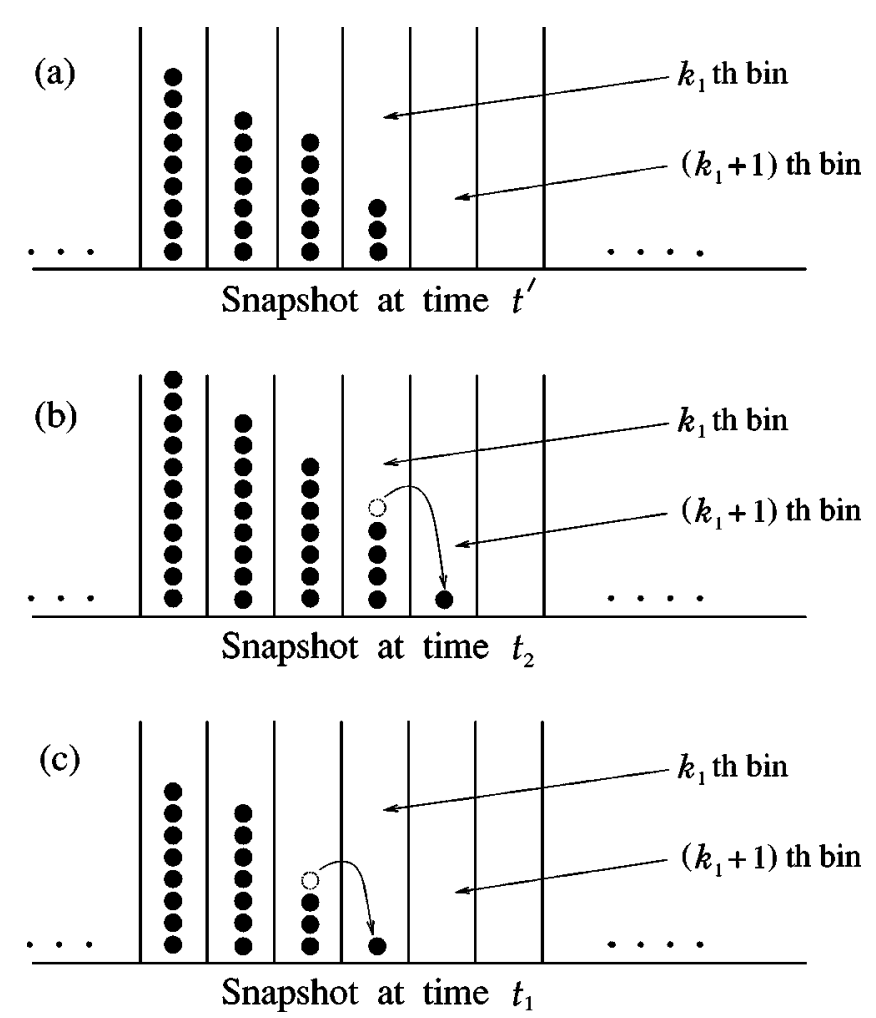

FIG. 1. Snapshots of one particular realization at times $t^{\prime}, t_{2}$, and $t_{1}$. The filled circles denote the $X$ particles in different bins. At time $t_{2}$, the $\left(k_{1}+1\right)$ th bin becomes the new foremost bin. In a similar manner, the $k_{1}$ th bin became the new foremost bin at time $t_{1}$.

For large $t-t_{0}, N_{t o t}(t)$ grows linearly and one may define the asymptotic front speed $v_{N}$ as

$$
v_{N}=\frac{1}{N_{t \rightarrow \infty}} \lim _{t \rightarrow \infty} \frac{N_{t o t}(t)-N_{t o t}\left(t_{0}\right)}{t-t_{0}} .
$$

Simultaneously, the position of the foremost bin also shifts towards the right. For long times, the average rate at which the position of the foremost bin shifts towards the right is the same as the front speed measured according to the definition, Eq. (4.2), as otherwise, an individual front realization will never reach an asymptotic shape.

Let us now examine the dynamics of the foremost bin in one particular realization. In Sec. III. The foremost bin moves towards the right by means of hops of the $X$ particles. The way this diffusion takes place is as follows: let us imagine that in one particular realization, at a certain time $t^{\prime}$, the index for the foremost bin is $k_{1}$, i.e., at time $t^{\prime}$, all the bins on the right of the $k_{1}$ th bin in that realization are not occupied by the $X$ particles [see Fig. 1(a)]. The diffusion of the $X$ particles from the $k_{1}$ th bin to the $\left(k_{1}+1\right)$ th bin is not a continuous process. As a result, it takes some more time before the first $X$ particle diffuses from the $k_{1}$ th bin to the $\left(k_{1}+1\right)$ th bin. Let us denote, by $t_{2}$, the time instant at which this diffusion takes place [see Fig. 1(b)]. Clearly, there is no exchange of $X$ particles between the $k_{1}$ th bin and the $\left(k_{1}\right.$ $+1)$ th bin in the time interval $t^{\prime} \leqslant t<t_{2}$. During this time, 
however, there can be time spans, where the number of the $X$ particles in the $k_{1}$ th bin may drop down to zero, since in the time interval $t^{\prime} \leqslant t<t_{2}$, the diffusion of the $X$ particles out of the $k_{1}$ th bin towards its left is an allowed process. By definition, at time $t_{2}$, the $\left(k_{1}+1\right)$ th bin becomes the "new foremost bin." Let us now denote, by $t_{1}$, the time instant when the $k_{1}$ th bin became the "new foremost bin" due to the diffusion of an $X$ particle from the $\left(k_{1}-1\right)$ th bin in exactly the same manner [see Fig. 1(c)]. In this notation, therefore, $t_{2}$ $>t_{1}$, and we say that $k_{1}$ th bin remains the foremost bin for the time interval $\Delta t=t_{2}-t_{1}$. If we now have a series of such $\Delta t$ values in sequence, i.e., a sequence of time values $\Delta t_{1}, \Delta t_{2}, \ldots, \Delta t_{j}$, for which a bin remains the foremost bin, then it is easily seen that the asymptotic front speed is also given by

$$
v_{N}=\lim _{j \rightarrow \infty} j\left[\sum_{j^{\prime}=1}^{j} \Delta t_{j^{\prime}}\right]^{-1} .
$$

Put in a different way, if we denote the probability that a foremost bin remains the foremost bin for time $\Delta t$ by $\mathcal{P}(\Delta t)$, the asymptotic front speed, according to Eq. (4.3), is given by

$$
v_{N}=\left[\int_{0}^{\infty} d(\Delta t) \Delta t \mathcal{P}(\Delta t)\right]^{-1} .
$$

Henceforth, our goal is to obtain a theoretical expression for $\mathcal{P}(\Delta t)$, for given parameter values $N$ and $\tilde{\gamma}$. As a first approach, we will make an attempt to devise a mean-field theory for this purpose. It is precisely at this place that we need to study the origin and the consequences of the breakdown of the definition of the comoving coordinate, $\xi$.

\section{A. The stalling phenomenon: Lowest order approach}

The origin of the breakdown of the definition of the comoving coordinate, $\xi$, in a mean-field description is quite easy to understand. As can be seen from the discussion in the paragraph above Eq. (4.3), the key lies in the fact that for the time a foremost bin remains the foremost bin, the front in the tip region does not move at all. We refer to this as the "stalling phenomenon." During such stalling periods, all the dynamics is confined within the left of (including) the foremost bin. It is this stalling phenomenon that is responsible for the breakdown of the definition of the comoving coordinate, $\xi$ [31].

Our first step in analyzing the stalling phenomenon is to get back to the $k$ and the $t$ coordinates, but in a different way than we have used them so far: the foremost bin, for the entire duration it remains the foremost bin, is indexed by an arbitrary fixed integer $k_{f}$ in this new scheme of relabeling the bin indices. The rest of the bins are accordingly indexed by their positions with respect to the $k_{f}$ th bin. Moreover, we start to count time (i.e., set the clock at $t=0$ ) as soon as an $X$ particle diffuses into the $k_{f}$ th bin from the left and stop the clock just when an $X$ particle diffuses from the $k_{f}$ th bin to the right. This relabeling strongly resembles the system of comoving coordinates, hence we call it the "quasi-comoving
TABLE I. Summary of the various coordinate labels used in the paper.

\begin{tabular}{c}
\hline $\begin{array}{c}\text { The label of the foremost bin between time } \\
t=0 \text { and } t=\Delta t \text { in an actual realization, } \\
\text { e.g., in a computer simulation. }\end{array}$ \\
$\begin{array}{c}\text { The label of the bin that attains the status of } \\
\text { the foremost bin at time } t=0 \text { in the mean-field } \\
\text { theory that we describe in this section. } \\
\text { Naturally, at } t=0, \text { the density of } X \text { particles }\end{array}$ \\
in it is equal to $1 / N$. \\
The label of the bin, where the average front \\
profile $\phi^{(0)}$, extrapolated from behind, is \\
equal to $1 / N$. \\
$k_{m_{0}} \quad \begin{array}{c}\text { The label of the bin behind the tip, from } \\
\text { which point on corrections to the profile } \phi^{(0)} \\
\text { are neglected. }\end{array}$ \\
The bin where $\phi^{(0)}$ becomes zero, i.e., the \\
$k_{n} \quad$ value of $k$ where the argument of the sin function \\
of $\phi^{(0)}$ becomes $\pi$.
\end{tabular}

coordinates." In this formulation, the clock stops at time $\Delta t$ and resets itself to zero. In this manner, the propagation of the front is a repetitive process of creating new foremost bins in intervals of $\Delta t$. Of course, it is a probabilistic process, in which the value of $\Delta t$ is not fixed.

Our mean-field theory essentially mimics the stalling phenomenon just as we see it in a computer simulation. In this theory, we also have a foremost bin, which we index by a fixed integer $k_{m}$ in the quasi-comoving frame. In these coordinates, we describe the dynamics of the front by the average number of $X$ particles in the bins. Between the times $t=0$ and $t=\Delta t$, all the dynamics of the front is confined to the left of (including) the $k_{m}$ th bin. For the benefit of the reader, we summarize the various coordinates $k$ used in this paper in Table I.

The equations of motion in this quasi-comoving frame, analogous to Eq. (2.5), in terms of the bin indices $k$ are therefore given by

$$
\begin{gathered}
\frac{\partial}{\partial t}\left\langle N_{k}(t)\right\rangle=\tilde{\gamma}\left[\left\langle N_{k+1}(t)\right\rangle+\left\langle N_{k-1}(t)\right\rangle-2\left\langle N_{k}(t)\right\rangle\right] \\
+\left\langle N_{k}(t)\right\rangle-\frac{1}{N}\left[\left\langle N_{k}^{2}(t)\right\rangle-\left\langle N_{k}(t)\right\rangle\right], \\
\forall k<k_{m}, \\
\frac{\partial}{\partial t}\left\langle N_{k}(t)\right\rangle=\tilde{\gamma}\left[\left\langle N_{k-1}(t)\right\rangle-\left\langle N_{k}(t)\right\rangle\right]+\left\langle N_{k}(t)\right\rangle \\
-\frac{1}{N}\left[\left\langle N_{k}^{2}(t)\right\rangle-\left\langle N_{k}(t)\right\rangle\right] \quad \text { for } k=k_{m},
\end{gathered}
$$

and

$$
\left\langle N_{k}\right\rangle=0, \quad \forall k>k_{m},
$$


for $0<t<\Delta t$, with the initial condition that $\left\langle N_{k_{m}}\right\rangle=N_{k_{m}}$ $=1$ at time $t=0$. The angular brackets above denote quantities averaged over many snapshots of one single front realization at time $t$. We focus our attention to the region at the leading edge of the front (up to the $k_{m}$ th bin), where the nonlinearities can be neglected so that the dynamics is given by

$$
\begin{gathered}
\frac{\partial}{\partial t} \phi_{k}(t)=\tilde{\gamma}\left[\phi_{k+1}(t)+\phi_{k-1}(t)-2 \phi_{k}(t)\right]+\phi_{k}(t), \\
\forall k<k_{m}, \\
\frac{\partial}{\partial t} \phi_{k}(t)=\tilde{\gamma}\left[\phi_{k-1}(t)-\phi_{k}(t)\right]+\phi_{k}(t) \quad \text { for } k=k_{m},
\end{gathered}
$$

with $\phi_{k}(t)=\left\langle N_{k}(t)\right\rangle / N, 0<t<\Delta t$ and $\phi_{k_{m}}=1 / N$ at time $t$ $=0$. Equation (4.5) explicitly illustrates that the growth of the probability ahead of the foremost bin is somewhat different from that behind the foremost bin as a result of the stalling.

Before, we already introduced the probability $\mathcal{P}(\Delta t)$ that the foremost bin remains the foremost one between the times $t=0$ and $t=\Delta t$. Since the foremost bin ceases to be the foremost one when a particle jumps out of it to the neighboring empty one on the right, $\mathcal{P}(t)$ obeys the equation

$$
\mathcal{P}(\Delta t)=\tilde{\gamma}\left\langle N_{k_{m}}(\Delta t)\right\rangle \exp \left[-\tilde{\gamma} \int_{0}^{\Delta t} d t\left\langle N_{k_{m}}(t)\right\rangle\right],
$$

satisfying the normalization condition. Clearly, as one can see from Eqs. (4.4) and (4.7), the proper asymptotic speed is determined by $\left\langle N_{k_{m}}(t)\right\rangle$, which in turn must come out of the solution of Eq. (4.6), i.e., from the effect of the stalling phenomenon on the leading edge of the front.

The dynamics of the leading edge of the front, described by our mean-field theory in the preceding two paragraphs, is a clear oversimplification. In an actual realization, the dynamics of the tip that governs the probability distribution $\mathcal{P}(\Delta t)$ in the quasi-comoving frame, is quite complicated. The foremost bin has only a few particles, and as a consequence, the fluctuation in the number of particles in it plays a very significant role in deciding the nature of the probability distribution $\mathcal{P}(\Delta t)$. Arising out of the fluctuations, there are two noteworthy events that have serious consequences for the behavior of $\mathcal{P}(\Delta t)$.

(i) The creation of the new foremost bins is a probabilistic process, for which the time scale is characterized by $1 / v_{N}$. However, if several foremost bins are created in a sequence relatively fast compared to the time scale set by $1 / v_{N}$, then one naturally expects that soon there would be a case when the new foremost bin would be created at an unusually large value of $\Delta t$.

(ii) According to our definition, in the actual realization of the system, the $k_{f}$ th bin remains the foremost bin between time $t=0$ and $t=\Delta t$. However, it may so happen that during this time, all the $X$ particles in the $k_{f}$ th bin diffuse back to the left, leaving it empty for some time, until some other $X$ particle hops into it, making it nonempty back again at a time $0<t<\Delta t$.

By the nature of construction, no mean-field theory can ever hope to capture the fullest extent of these fluctuations, and the one that we just presented above [that represents the effect of the stalling phenomenon on the asymptotic speed selection mechanism for the front by considering $\mathcal{P}(\Delta t)]$, is no exception. Therefore, in this mean-field theory that we described in this section, such fluctuation effects are completely suppressed. We will return to these fluctuation effects in Sec. IV C below, where we will make an attempt to estimate the effects of these fluctuations on $\mathcal{P}(\Delta t)$. The corresponding estimates will then be used to improve the theoretical prediction of $\mathcal{P}(\Delta t)$ as well as to draw limits on the validity of our mean-field theory.

\section{B. Effect of the stalling phenomenon on the front shape near the foremost bin}

In the preceding subsection, we obtained a mean-field type expression for $\mathcal{P}(\Delta t)$ in terms of $\left\langle N_{k_{m}}(t)\right\rangle$. A first approximation for $\left\langle N_{k_{m}}(t)\right\rangle$ would be obtained from the solution of Eq. (4.5) above. However, in practice, the average occupation $\left\langle N_{k_{m}}(t)\right\rangle$ is affected by the stalling effect itself. We now account for this effect in a self-consistent way by calculating the corrections to the front shape near the foremost bin. We start with Eq. (4.6), and subsequently build upon the considerations of Sec. III, where we derived the solution $\phi(\xi)=A \sin \left[z_{i} \xi\right] \exp \left(-z_{r} \xi\right)$ at the leading edge of the front.

A naive approach would be to claim that the shape of the leading edge of the front, described by the set of equations (4.6), is given by $\phi_{k}(t)=A \sin \left[z_{i}\left(k-v_{N} t\right)+\beta\right] \exp \left[-z_{r}(k\right.$ $\left.\left.-v_{N} t\right)\right]$ for $0<t<\Delta t$ in the quasi-comoving frame. Notice that we have reintroduced the phase factor $\beta$ inside the argument of the sine function, in view of the fact that $k$ can only take integral values. This solution of $\phi_{k}(t)$ would once again generate the same dispersion relation as in Eq. (A2). However, it is intuitively quite clear that this solution of $\phi_{k}(t)$ cannot hold all the way upto $k=k_{m}$, since the equations of motion for $k<k_{m}$ are different from the equation of motion for $k=k_{m}$. First of all, $\phi_{k_{m}}(t=0)=1 / N$, which may not necessarily be equal to the value of the function $A \sin \left[z_{i}\left(k_{m}-v_{N} t\right)+\beta\right] \exp \left[-z_{r}\left(k_{m}-v_{N} t\right)\right]$ at time $t=0$. Second, for the entire duration of $0<t<\Delta t$, the tip of the front is stationary at $k_{m}$, and as a result, the flow of particles from the left starts to accumulate in the $k_{m}$ th (foremost) bin. With increasing value of $t$, bins on the left of the foremost bin get to know that the tip of the front has stalled, and the correlation among different bins starts to develop on the left of the foremost bin. As a result, an excess of particle density beyond the corresponding "normal solution" values $A \sin \left[z_{i}(k\right.$ $\left.\left.-v_{N} t\right)+\beta\right] \exp \left[-z_{r}\left(k-v_{N} t\right)\right]$ builds up on the left of (including) the foremost bin over time. This is demonstrated in Fig. 2.

To deal with the effect of stalling phenomenon on the density of $X$ particles in the bins at the tip of the front, which 


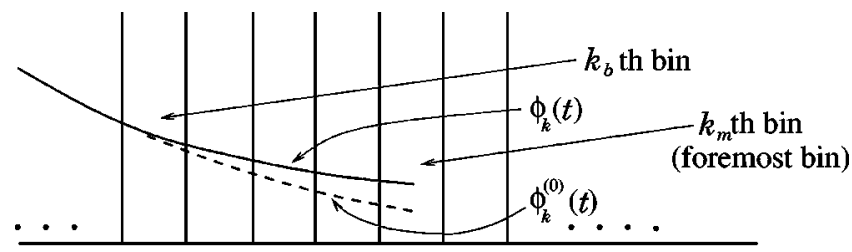

Snapshot at time $t$

FIG. 2. Snapshot of the tip of the front in a mean-field description at time $0<t<\Delta t$, showing density buildup of $X$ particles on and behind the foremost bin for a large enough value of $t$. The dotted curve is for the "normal solution," $\phi_{k}^{(0)}(t)=A \sin \left[z_{i}(k\right.$ $\left.\left.-v_{N} t\right)+\beta\right] \exp \left[-z_{r}\left(k-v_{N} t\right)\right]$. The solid curve is for the actual function $\phi_{k}(t)$. Even though both $\phi_{k}^{(0)}(t)$ and $\phi_{k}(t)$ are discrete functions of $k$, we have drawn continuous curves for clarity.

is very crucial to calculate $\left\langle N_{k_{m}}(t)\right\rangle$, let us express $\phi_{k}(t)$ as

$$
\phi_{k}(t)=\phi_{k}^{(0)}(t)+\delta \phi_{k}(t),
$$

where $\phi_{k}^{(0)}(t)=A \sin \left[z_{i}\left(k-v_{N} t\right)+\beta\right] \exp \left[-z_{r}\left(k-v_{N} t\right)\right]$. The quantity $\delta \phi_{k}(t)$ then denotes the deviation of the density of the $X$ particles in the $k$ th bin from the "normal solution" $\phi_{k}^{(0)}(t)$. It takes time for the deviation to develop in any bin, and moreover, since such correlation effects spread diffusively, the information that the tip of the front has stalled at the foremost bin does not affect too many bins behind the foremost bin. Thus, it is reasonable to assume that on the left of the foremost bin, there exists a bin, henceforth indexed by $k_{b}$ in this quasi-comoving coordinate (i.e., $k_{b}<k_{m}$ ), where the magnitude of $\delta \phi_{k}(t)$ is so small that we can impose the condition that $\delta \phi_{k_{b}}(t)=0$. We then substitute Eq. (4.8) in Eq. (4.6) and without having to worry about the equation of motion for $\delta \phi_{k_{b}}(t)$, we obtain the equations of motion of the quantities $\delta \phi_{k}(t)$ for $k_{b}<k \leqslant k_{m}$ as

$$
\begin{gathered}
\frac{\partial}{\partial t} \delta \phi_{k}(t)=\tilde{\gamma}\left[\delta \phi_{k+1}(t)-2 \delta \phi_{k}(t)\right]+\delta \phi_{k}(t) \\
\text { for } k=k_{b}+1 \\
\frac{\partial}{\partial t} \delta \phi_{k}(t)=\tilde{\gamma}\left[\delta \phi_{k+1}(t)+\delta \phi_{k-1}(t)-2 \delta \phi_{k}(t)\right] \\
+\delta \phi_{k}(t), \quad \forall\left(k_{b}+1\right)<k<k_{m}, \\
\frac{\partial}{\partial t} \delta \phi_{k}(t)=\tilde{\gamma}\left[\delta \phi_{k-1}(t)-\delta \phi_{k}(t)\right]+\delta \phi_{k}(t) \\
-\tilde{\gamma}\left[\phi_{k+1}^{(0)}-\phi_{k}^{(0)}\right] \\
\text { for } k=k_{m} .
\end{gathered}
$$

If we now denote the $\left(k_{m}-k_{b}\right)$-dimensional column vector $\left[\delta \phi_{k_{m}}(t), \delta \phi_{k_{m}-1}(t), \ldots, \delta \phi_{k_{b}+1}(t)\right]$ by $\delta \Phi(t)$, then Eq. (4.9) becomes an inhomogeneous linear differential equation in $\delta \Phi(t)$, given by

$$
\frac{d}{d t} \delta \Phi(t)=\mathbf{M} \delta \Phi(t)+\delta \Phi_{p},
$$

where $\mathbf{M}$ is the $\left(k_{m}-k_{b}\right) \times\left(k_{m}-k_{b}\right)$-dimensional tridiagonal symmetric matrix,

$$
\mathbf{M}=\left[\begin{array}{cccccc}
1-\tilde{\gamma} & \tilde{\gamma} & 0 & \ldots & 0 & 0 \\
\tilde{\gamma} & 1-2 \tilde{\gamma} & \tilde{\gamma} & 0 & \ldots & 0 \\
0 & \tilde{\gamma} & 1-2 \tilde{\gamma} & \tilde{\gamma} & \ldots & 0 \\
\cdot & \cdot & \cdot & . & . & . \\
0 & \ldots & 0 & \tilde{\gamma} & 1-2 \tilde{\gamma} & \tilde{\gamma} \\
0 & \ldots & 0 & 0 & \tilde{\gamma} & 1-2 \tilde{\gamma}
\end{array}\right],
$$

and $\delta \Phi_{p}=\left[\tilde{\gamma}\left(\phi_{k_{m}}^{(0)}-\phi_{k_{m}+1}^{(0)}\right), 0, \ldots, 0\right]$. The solution of the linear inhomogeneous differential equation, Eq. (4.10), is straightforwardly obtained as

$$
\begin{aligned}
\delta \Phi(t)= & \exp [\mathbf{M} t] \delta \Phi(t=0) \\
& +\int_{0}^{t} d t^{\prime} \exp \left[\mathbf{M}\left(t-t^{\prime}\right)\right] \delta \Phi_{p}\left(t^{\prime}\right) .
\end{aligned}
$$

To obtain the expression of $\left\langle N_{k_{m}}(t)\right\rangle$, which is our final goal, we have to determine the unknowns $\delta \Phi(t=0)$. Of these, the expression of $\delta \phi_{k_{m}}(t=0)$ is already known from the fact that at time $t=0$, there is exactly one $X$ particle in the $k_{m}$ th bin, i.e.,

$$
\delta \phi_{k_{m}}(t=0)=\frac{1}{N}-\phi_{k_{m}}^{(0)}(t=0) .
$$

The values of $\delta \phi_{k}(t=0)$ for $k_{b}<k<k_{m}$ are also quite easily determined when we notice that at time $t=\Delta t$, the values of $\delta \phi_{k}(t=\Delta t)$ must reach the corresponding values of $\delta \phi_{k-1}(t=0)$, because the average shape of the front repeats itself once every $\Delta t$ time (note here that the repetitive character of foremost bin creation in the quasi-comoving frame is built in). This leads us to the following set of $k_{m}-k_{b}-1$ consistency conditions:

$$
\begin{gathered}
\delta \phi_{k_{b}+1}(t=0)=\int_{0}^{\infty} d(\Delta t) \mathcal{P}(\Delta t) \delta \phi_{k_{b}+2}(\Delta t), \\
\vdots \\
\delta \phi_{k_{m}-2}(t=0)=\int_{0}^{\infty} d(\Delta t) \mathcal{P}(\Delta t) \delta \phi_{k_{m}-1}(\Delta t), \\
\delta \phi_{k_{m}-1}(t=0)=\int_{0}^{\infty} d(\Delta t) \mathcal{P}(\Delta t) \delta \phi_{k_{m}}(\Delta t)-\frac{1}{N} .
\end{gathered}
$$

The equation for $\delta \phi_{k_{m}-1}(\Delta t)$ is different from the other ones in Eq. (4.14), as it has an extra $-1 / N$ on its right-hand 
side (rhs). This is so, because the one $X$ particle that hopped over to the $k_{m}$ th bin at $t=0$, came from the $\left(k_{m}-1\right)$ th bin.

In actuality, Eq. (4.14) should be written in terms of $\phi_{k}$ 's. If we do so, then on the rhs of the corresponding equations, we have integrals of the form $\int_{0}^{\infty} d(\Delta t) \mathcal{P}(\Delta t) \phi_{k}^{(0)}(\Delta t)$. We have replaced these integrals by $\phi_{k-1}^{(0)}(t=0)$. This is consistent with the fact that in an average sense, the underlying particle density field $\phi_{k}^{(0)}(t)$ has a uniformly translating solution. The leftover $\delta \phi_{k}$ terms then yield Eq. (4.14).

In terms of this formulation, the leading edge of the front, whose equation of motion is governed by the linearized equation, Eq. (4.6), is divided into two parts [32]. In the first part, which lies on the left of (including) the $k_{b}$ th bin, the solution is given by the form $\phi_{k}(t)=A \sin \left[z_{i}\left(k_{m}-v_{N} t\right)\right.$ $+\beta] \exp \left[-z_{r}\left(k_{m}-v_{N} t\right)\right]$ for $0<t<\Delta t$. In the second part, constituted by the bins indexed by $k$, such that $k_{b}<k \leqslant k_{m}$, the shape of front is given by Eqs. (4.7)-(4.14). The first part yields the linear dispersion relation, Eq. (A2), while the second part yields more complicated and nonlinear relations between $v_{N}, z_{r}$, and $z_{i}$ involving several other unknown quantities as a self-consistent set of equations. With the values of $A, k_{b}$, and $k_{m}$ externally determined, if one counts the number of equations and the number of unknowns that are available at this juncture for the selected asymptotic speed $v_{N}$, then, from Eqs. (A2), (4.4), (4.8), and (4.12)-(4.14), it is easy to see that they involve as many unknowns as the number of equations. The value of $A$ is obtained by matching the mean-field solution of the bulk of the front, where the nonlinearities of Eq. (4.5) play a significant role, with the solution of the leading edge of the front described by the linear equations [i.e., Eq. (4.6)]. On the other hand, obtaining the value of $k_{b}$ and $k_{m}$, for a given set of parameters $N$ and $\tilde{\gamma}$, is a more complicated process and now we address it in the next few paragraphs. We will take up these issues in further detail in Sec. V D as well, when we compare our theoretical results with the results obtained from the computer simulation.

While it is easy to determine the foremost bin and hence define $k_{f}$ for any given realization in a computer simulation, the question how to obtain the values of $k_{m}, \beta$, and $k_{b}$ for a given set of values of $N$ and $\tilde{\gamma}$, still remains to be answered. As a first step to answer this question, we redefine $A$ and absorb the quantity $\beta$ in $k$ by a change of variable, $z_{i} k+\beta$ $\rightarrow z_{i} k$, such that in the quasi-comoving frame, $\phi_{k}^{(0)}(t)$ reduces to $A \sin \left[z_{i}\left(k-v_{N} t\right)\right] \exp \left[-z_{r}\left(k-v_{N} t\right)\right]$. First, this makes $k$ a continuous variable as opposed to a discrete integral one. Second, the number of unknown quantities is also reduced from three to two, namely, to $k_{m}$ and $k_{b}$.

If we now look back at Fig. 2, and recapitulate the structure of the mean-field theory we presented in this section, we realize that the buildup of particles in the bins at the tip of the front due to the stalling phenomenon always makes the curve $\phi_{k}(t=0)$ lie above $\phi_{k}^{(0)}(t=0)$, when they are plotted against the continuous variable $k$. In our mean-field theory, $\phi_{k_{m}}(t=0)=1 / N$, which clearly means that $\phi_{k_{m}}^{(0)}(t=0)$ $<1 / N$ and since $\phi_{k}^{(0)}(t=0)$ is a monotonically decreasing function of $k$, this further implies that $k_{m}>k_{m_{0}}$, where $\phi_{k_{m_{0}}}^{(0)}(t=0)=1 / N$.
In our mean-field theory, what is the numerical value of $\left(k_{m}-k_{m_{0}}\right)$, the distance between the bin, where the lowest order approximation $\phi^{(0)}$ reaches the values $1 / N$ and the bin, where the actual average profile $\phi$ reaches this value? For arbitrary values of $N$ and $\tilde{\gamma}$, this is not an easy question to answer.

To check our theory, in this paper we confine ourselves mostly to the case of $\tilde{\gamma}=$ (growth rate) $=1$, as it is the most illustrative case to demonstrates the multiple facets of fluctuating front propagation. For a part of the analysis, we also consider the $\tilde{\gamma}=0.1$ case. For such values of $\gamma$, i.e., if $\tilde{\gamma}$ is too small $(\tilde{\gamma} \ll 1)$, or not too large $(\tilde{\gamma} \sim 1)$, the only information that we have at our disposal to obtain the value of the continuous parameter $k_{m}$, is the fact that $k_{m}>k_{m_{0}}$. For such values of $\tilde{\gamma}$, therefore, the only remaining way to generate the $\mathcal{P}(\Delta t)$ curve is to use trial values of $k_{m}$, for $k_{m}>k_{m_{0}}$ in an iterative manner [33] [recall that the value of $k_{m}$ is needed for the initial condition, Eq. (4.13)]. For such values of $\tilde{\gamma}$, the use of the trial values of $k_{m}$ to generate $\mathcal{P}(\Delta t)$ also requires the value of $k_{m}-k_{b}$ as an external parameter, which can be chosen to be a few, say $\sim 4$ [of course, this number can be increased to obtain higher degree of accuracy for the $\delta \phi_{k}(t=0)$ values]. We will take up further details about it in Sec. V. However, before that, we next discuss two additional fluctuation effects that have important consequences on the $\mathcal{P}(\Delta t)$ curve. We also mention here that we have explored the possibility of a relation between $k_{f}$, obtained from computer simulation results, and $k_{m}$, but due to the fact that $k_{f}$ has stochastic fluctuations in time, such a relation does not exist.

\section{Additional fluctuation effects}

Having described the mean-field theory, we are now in a position to assess its accuracy or validity for the probability distribution $\mathcal{P}(\Delta t)$ that it generates, before we start to look for numerical confirmation. At the end of Sec. IV A, we have mentioned that the fluctuation of the number of $X$ particles in the foremost bin plays a very significant role in deciding the nature of $\mathcal{P}(\Delta t)$. Such fluctuations are not captured in our mean-field theory, which simply assumes that the number of $X$ particles in the foremost bin at $t=0$ is 1 and afterwards the number of the $X$ particles in it increases through the process of a mean growth. In particular, at the end of Sec. IV A we have described two kinds of events that, we now argue, affect the nature of $\mathcal{P}(\Delta t)$ for large values of $\Delta t$, compared to the time scale set by $1 / v_{N}$.

\section{Few foremost bins are created too fast in a sequence}

The first of these events is that if a few of the new foremost bins are created relatively fast in a row, then soon there would be a case of a new foremost bin creation that takes an unusually long time. Naturally, this gives $\mathcal{P}(\Delta t)$ a higher value than what our mean-field theory does for large values of $\Delta t$. The reason for this is quite simple: the mean growth of the number of $X$ particles in the foremost bin is exponential in time, which would indicate that if one describes the 
growth of the number of $X$ particles in the foremost bin simply by mean growth, then the probability distribution $\mathcal{P}(\Delta t)$ decreases very rapidly for large $\Delta t$, and clearly that fails to describe the slow decay of $\mathcal{P}(\Delta t)$ for large $\Delta t$ arising out of this event.

Unfortunately, there is no way to estimate the effect of this event within the scope of any mean-field theory, since by its sheer nature, it can only be described by the multitime correlation functions of the times required for sequential creations of new foremost bins. For this reason, we call this event "correlated diffusion event" for later reference. But the physical effect of it can be expressed in a slightly different manner which is more conducive for understanding the conditions of applicability of our mean-field theory. In our mean-field theoretical description, before a new foremost bin is created, the shape of the front is always the same mean shape, described by Eqs. (4.5). On the other hand, if a few of the new foremost bins are created relatively fast in a row, the leading edge of the front gets more and more elongated while the number of particles inside the bins in the leading edge does not get a chance to grow accordingly. Thus, this event creates significant deviation for the actual front shape from the front shape described by our mean-field theory. The magnitude of this deviation, measured by subtracting the mean-field density of the $X$ particles from the actual density of $X$ particles inside the bins at the leading edge of the front, is obviously negative. If we combine this argument with the fact that on an average, the probability of a new foremost bin creation increases with the increasing number of $X$ particles in the foremost bin, then it is easy to realize that after a sequence of such fast creations of new foremost bins, the front needs to replenish the number of $X$ particles in the leading edge before another new foremost bin is created. It is this replenishing process which is responsible for the next new foremost bin creation at a relatively long time.

It is now intuitively clear that in terms of the front shape, the larger the deviation such an event causes, the more $\mathcal{P}(\Delta t)$ will be affected for large values of $\Delta t$. Based on this, we now argue that for a fixed value of $N$, such an event does not affect the large $\Delta t$ behavior of $\mathcal{P}(\Delta t)$ curve for large values of $\tilde{\gamma}$ as much as it does for small values of $\tilde{\gamma}$. To reach this conclusion, one simply needs to observe the following: the mean shape and the corresponding density of the $X$ particles in the bins at the leading edge of the front is characterized by $z_{r}$ and $z_{i}$, and for small $\tilde{\gamma}$, the values of $z_{r}$ and $z_{i}$ is large and vice versa [as $z_{i} \sim z_{r} \sim \tilde{\gamma}^{-1 / 2}$, see Eq. (A9)]. For large $\tilde{\gamma}$, therefore, for the mean shape of the front, the leading edge is already quite elongated and the density of the $X$ particles at the tip of the front is quite small, compared to their small $\tilde{\gamma}$ values. As a result, for small $\tilde{\gamma}$, the magnitude of the deviation from the mean front shape, caused by such an event is much larger, and since the growth rate is always unity for all $\tilde{\gamma}$, it takes a much longer time to replenish the density of $X$ particles for small $\tilde{\gamma}$ than for large $\tilde{\gamma}$ values.

\section{Particles in the foremost bin jump back}

The other kind of fluctuation effect has to do with the fact that albeit according to our definition, the foremost bin re- mains the foremost one until time $t=\Delta t$, it may so happen that at some nonzero value of $t$, all the $X$ particles diffuse back to the left leaving the foremost bin empty for some time and then another $X$ particle diffuses into the foremost bin from the left, making it nonempty again at a finite value of $t$, say at $t=t_{0}$. Clearly, this event is much more unlikely to take place once the number of $X$ particles in the foremost bin has grown, since in that case, all the $X$ particles in the foremost bin have to diffuse back to the left. Essentially, this event is therefore restricted to the following sequence: (a) starting $t$ $=0$, the foremost bin remains occupied by a single $X$ particle for some time, (b) this $X$ particle then diffuses back to the left leaving the foremost bin empty, until (c) another $X$ particle diffuses into the foremost bin, making it nonempty again at $t=t_{0}$. Of course, the value of $t_{0}$ is not fixed and it is chosen probabilistically. For later reference, we call this "the vacant foremost bin event," and this event is much more likely to take place for large diffusion coefficient $\tilde{\gamma}$.

Based on the picture described in (a)-(c) in the preceding paragraph, we can now make a quantitative estimate of this particular event and accordingly correct the expression of $\mathcal{P}(\Delta t)$. One simply has to realize that if this event takes place, then the time at which the theory for mean growth of $X$ particles in the foremost bin (with exactly one $X$ particle to start with) can be applied in this quasi-comoving frame, shifts from $t=0$ to $t=t_{0}$. However, we also need to obtain an estimate for the value of $t_{0}$. This can be obtained using the following argument: if in step (b), the only $X$ particle in the foremost bin had diffused to the right, instead of diffusing to the left, it would have been a case of a new foremost bin creation, the time scale for which is set by $1 / v_{N}$. Since the probability of this single $X$ particle in the foremost bin to jump to the right is the same as the probability of it to jump to the left, we can also say that the time it takes for the $X$ particle in the foremost bin to diffuse back to the left takes approximately a time $1 / v_{N}$ starting $t=0$. Similarly, step (c) is exactly the same step as a "new foremost bin creation." Hence, after step (b) is over, it takes a further $1 / v_{N}$ time [34] for another $X$ particle to diffuse from the left into the foremost bin. Together, these two events make $t_{0} \approx 2 / v_{N}$ [35], and this argument illustrates that this event affects the behavior of $\mathcal{P}(\Delta t)$ only for $\Delta t \gtrsim 2 / v_{N}$. Having neglected the effect of the correlated diffusion events on the $\mathcal{P}(\Delta t)$ curve for $t$ $>2 / v_{N}$ (for which we have no theoretical estimate anyway), if we now claim that for all $\Delta t$ values greater than $2 / v_{N}$, the population of the $X$ particles in the foremost bin is described by Eqs. (4.5), but with the condition that $\left\langle N_{k_{m}}\left(t=t_{0}\right)\right\rangle$ $=N_{k_{m}}\left(t=t_{0}\right)=1 / N$, as opposed to having $\left\langle N_{k_{m}}(t=0)\right\rangle$ $=N_{k_{m}}(t=0)=1 / N$, then we can still incorporate the effect of this event (that arises out of fluctuations) within the scope of the mean-field theory that we described in this section. If this procedure is correct, then while comparing the theoretical $\mathcal{P}(\Delta t)$ curve with the $\mathcal{P}(\Delta t)$ curve obtained from the simulations, one would notice that for large values of $\Delta t$, this procedure underestimates the magnitude of $\mathcal{P}(\Delta t)$. Hitherto, this underestimation then would be an indication of the effect of the correlated diffusion events on $\mathcal{P}(\Delta t)$. We will return 
to these points once again in the following section, where we seek numerical confirmation of our theory [36].

\section{Summary of the status of the present approach and additional observations}

The discussion above completes the theoretical formulation for the asymptotic speed selection of the front. Before we discuss how given values of $N$ and $\tilde{\gamma}$ would generate the corresponding values of $v_{N}$ from our mean-field theory described in this section, we summarize our claims here and make a number of additional observations.

(1) Based upon the microscopic description of the front movement, we have formulated a mean-field theory that describes, on a lattice, the front propagation as a sequence of "halt-and-go" process. In this way of looking at the front propagation, essentially the number of $X$ particles at the tip of the front determines the asymptotic speed of the front. Since the number of $X$ particles at the tip of the front are rather few, the fluctuations in the number of $X$ particles at the tip of the front affects the asymptotic speed of the front in a strong manner. Part of the fluctuation effects can be estimated within the scope of this mean-field theory itself. The other part, for which the fluctuations can only be studied by means of a multitime correlation functions, is expected to affect the accuracy of our theory much more for small $\tilde{\gamma}$ than for large $\tilde{\gamma}$ values. Therefore, overall, in terms of numerical confirmation, one can expect to find a greater accuracy for large values of $\tilde{\gamma}$.

Moreover, for large $\tilde{\gamma}$, the discreteness of the lattice effects are suppressed, and therefore, for a given value of $N$, one would expect that the relative correction for the asymptotic front speed, $\left(v^{*}-v_{N}\right) / v^{*}$, must become small.

(2) There are two important aspects that one must take notice of. First, in a mean-field description that incorporates the effect of the stalling phenomenon, we have demonstrated from the microscopic dynamics that there exists a cutoff of particle density, which is expressed by the fact that in this mean-field description, $\phi_{k}(t)=0$ for $k>k_{m}$ against a finite value at $k=k_{m}$. Second, we have also demonstrated that the quantity $a$ in Sec. III is indeed an effective quantity only, as the solution of the linearized equation of the front, given by Eq. (3.3), is not valid near the foremost bin, and the fact that the asymptotic speed selection mechanism arises from a proper probabilistic description of the tip of the front.

(3) For very small values of $\tilde{\gamma}$, we have previously noticed that the correlated diffusion event plays a very dominant role that no mean-field theory can ever generate, so we should leave the $\tilde{\gamma} \ll 1$ case outside the purview of our meanfield theory (we will demonstrate this in the following section).

(4) To judge the appropriateness of our mean-field theory, as far as the generation of the numerical value of the asymptotic front speed for given values of $N$ and $\tilde{\gamma}$ is concerned, we make the following observations: (i) the case of $\tilde{\gamma} \ll 1$ cannot be studied in terms of a mean-field theory, (ii) the case of $\tilde{\gamma} \sim 1$ needs a trial value of $k_{m}>k_{m_{0}}$ and the use of a recursive feedback mechanism to generate the $\mathcal{P}(\Delta t)$ curve, and (iii) we still need the values of $A, z_{r}$, and $z_{i}$, which can be obtained only from the simulation data for given values of $N$ and $\tilde{\gamma}$. In view of these points, it is clear that this theory is unable to make a definitive prediction for $v_{N}$, without any assistance from the computer simulations whatsoever. Moreover, the Eqs. (4.4)-(4.14), which one needs to solve to generate the $\mathcal{P}(\Delta t)$ curve, are highly nonlinear equations, hence, this theory can only hope to show consistency with the results of the computer simulations, as opposed to produce a numerical value of $v_{N}$ which is then subsequently confirmed by the computer simulations.

(5) Finally, we note that unlike Eq. (3.4), this theory does not make the effect of the value of $N$ on the asymptotic front speed explicit. However, it is natural to expect that the effect of stalling of the front and the associated particle density buildup at the tip of the front on the front shape and speed would become less and less for increasing $N$. This would reflect in the comparison of the $\delta \phi_{k}(t=0)$ values against the corresponding $\phi_{k}^{(0)}(t=0)$ values in the bins at the very tip of the front. We would return to this point in Sec. V D 2.

\section{TEST OF THE THEORY AGAINST COMPUTER SIMULATIONS}

We now check our theory, as it has been presented in Secs. III and IV, against the results of the computer simulations. There have been quite a few aspects of the theory that we have presented in Secs. III and IV; and for a given set of values of $N$ and $\tilde{\gamma}$, testing all these aspects of our theory is not a short and easy process. To explain how we do the simulations, obtain $v_{N}$ and $A$, and check the front shape, we choose one particular set of $N$ and $\tilde{\gamma}$ values, namely, $N$ $=10^{4}$ and $\tilde{\gamma}=1$. We then use these methods to obtain the simulation data for three other values of $N$, namely, $N$ $=10^{2}, N=10^{3}$, and $N=10^{5}$, keeping the value of $\tilde{\gamma}$ fixed at 1. Based on this scheme, this section is divided into five subsections. In Sec. V A, we present the simulation algorithm and obtain $v_{N}$ for $\tilde{\gamma}=1$ and $N=10^{4}$. In Sec. V B, we summarize the method to calculate $A$, and subsequently obtain its value for $\tilde{\gamma}=1$ and $N=10^{4}$ using the results of Sec. $\mathrm{V}$ A. In the Sec. V C, we contrast the simulation results of Secs. V A and V B with the theory of Sec. III. In Sec. V D, we test our theoretical predictions for $\mathcal{P}(\Delta t)$ against the computer simulation results for $N=10^{4} 10^{2}, 10^{3}$, and $10^{5}$, and $\tilde{\gamma}=1$ (in that order). Moreover, in Sec. IV, we have conjectured that the mean-field theory mimicking the stalling phenomenon would be less successful for small values of $\tilde{\gamma}$. We verify this conjecture in Sec. V D 2 by means of a relative comparison of the theoretical and simulation $\mathcal{P}(\Delta t)$ curves for $\tilde{\gamma}=0.1$ and $N=10^{4}$. We also remind the reader that in Secs. V A-V C, $k$ and $t$, respectively, denote the laboratory bin coordinate and actual physical time (and therefore they do not relate to the quasi-comoving coordinates or the resetting of clocks that requires $0<t<\Delta t$ ). 


\section{A. Computer simulation algorithm}

Our algorithm for carrying out the computer simulations is the same as it has been described in Ref. [6]. The starting density profile of the $X$ particles is a step function, given by $\phi_{k}(t=0)=\left[1-\Theta\left(k-k_{0}\right)\right]$, for some $k_{0}$. The simulation algorithm consists of repetitive iterations of two basic steps.

(i) Let us assume that at any time $t$, the configuration of the system is given by $\left(N_{1}, N_{2}, \ldots, N_{k^{\prime}}\right)$, for some $k^{\prime}$. The total rate of possible transitions, $W_{k}$, for the $N_{k}$ number of $X$ particles in the $k$ th bin are the sum of $2 N_{k}$ diffusions, creation of $N_{k}$ new $X$ particles, and annihilation of $N_{k}\left(N_{k}\right.$ $-1) / N$ number of $X$ particles, i.e.,

$$
W_{k}=2 \tilde{\gamma} N_{k}+N_{k}+\frac{N_{k}\left(N_{k}-1\right)}{N} .
$$

The total rate of transition, $W_{t o t}$, for all the bins is therefore

$$
W_{\text {tot }}=\sum_{k=1}^{k^{\prime}} W_{k}
$$

Starting at time $t$, the probability of no transition happening for an interval $\tau$ is given by

$$
\wp(\tau)=\exp \left(-W_{\text {tot }} \tau\right)
$$

Before any transition takes place, a random number $r_{0}$ is chosen within the interval $[0,1)$. The time $\tau$ that one needs to wait before any transition happens is then determined as

$$
\tau=-\frac{1}{W_{t o t}} \ln r_{0}
$$

(ii) With the time $\tau$ for a transition at our disposal, the bin where the transition takes place and the specific transition in that bin must also be determined. To do so, we choose another set of two random numbers, $r_{1}$ and $r_{2}$, in $[0,1)$. From the numerical value of $r_{1}$ and the fact that the probability of a transition taking place in the $k$ th bin is given by $W_{k} / W_{\text {tot }}$, we determine the index of the bin where the transition takes place. Similarly, the particular transition in the $k$ th bin is determined from the numerical value of $r_{2}$ and considering the probabilities of different kinds of transitions in the $k$ th bin,

$($ probability of a diffusion to the right $)=\frac{\tilde{\gamma} N_{k}}{W_{k}}$, $($ probability of a diffusion to the left $)=\frac{\tilde{\gamma} N_{k}}{W_{k}}$, $($ probability of breeding a new $X$ particle $)=\frac{N_{k}}{W_{k}}$,

(probability of annihilating an $X$ particle $)=\frac{N_{k}\left(N_{k}-1\right)}{N W_{k}}$.

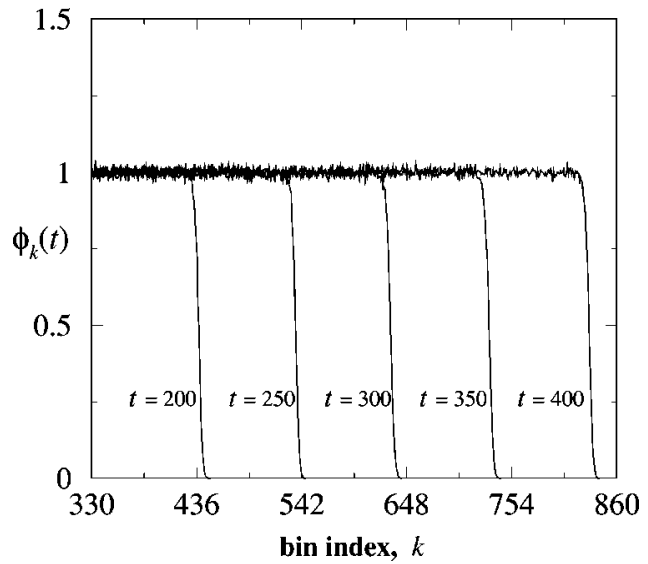

FIG. 3. To illustrate that the front reaches its steady state shape before $t=200$, the plot of $\phi_{k}(t)$ vs $t$ for five different values of $t$ spaced at regular intervals, $t=200, t=250, t=300, t=350$, and $t$ $=400$, are shown above.

Once the transition is determined, the configuration of the system is subsequently updated. However, any $X$ particle diffusing from the first bin (i.e., $k=1$ ) towards the left is immediately replaced.

In this subsection, we focus on one particular set of values of $\tilde{\gamma}$ and $N$, namely, $\tilde{\gamma}=1$ and $N=10^{4}$. The value of $k_{0}$ for the initial density profile of the $X$ particles is chosen to be 50 . Starting at $t=0$, we let this initial profile evolve in time. To obtain the random numbers, we use the random number generator drand48 provided in the standard C library functions with the initial seed [37] $s=123456$. It turns out that to a very good approximation, the front shape reaches a steady state somewhere before $t=200$. The front shapes from $t$ $=200$ to $t=400$ is shown in Fig. 3 as an illustration. For measurement of the asymptotic quantities, therefore, we take $t=200$ as our starting point.

To calculate the asymptotic speed of the front, we measure the $\Delta t$ values for creating new foremost bins after $t$ $=200$ till $t=700$. We find that altogether there are 980 different $\Delta t$ values in this time interval. Assuming that the $j$ th value of $\Delta t$ takes place at time $t_{j}(j=1,2, \ldots, 980)$, we define the $j$ th cumulative average of the $\Delta t$ values as

$$
\langle\Delta t\rangle_{j}=\frac{1}{j} \sum_{j^{\prime}=1}^{j} \Delta t_{j^{\prime}},
$$

which subsequently allows us to define the speed at time $t_{j}$ as

$$
v_{N}\left(t_{j}\right)=\frac{1}{\langle\Delta t\rangle_{j}} .
$$

Naturally, for small values of $j$, the values of $v_{N}\left(t_{j}\right)$ fluctuate, but as $j$ becomes large, the fluctuations die out and $v_{N}\left(t_{j}\right)$ approaches $v_{N}$. The plot of $v_{N}\left(t_{j}\right)$ vs $t_{j}$ is shown in Fig. 4 for $j=1,2, \ldots, 980, t_{1}=200.562$ and $t_{980}=699.271$. We notice from the plot that the fluctuations in $v_{N}\left(t_{j}\right)$ are really small for $t_{j}>500$. The $v_{N}\left(t_{j}\right)$ values for $t_{j}>500$, 


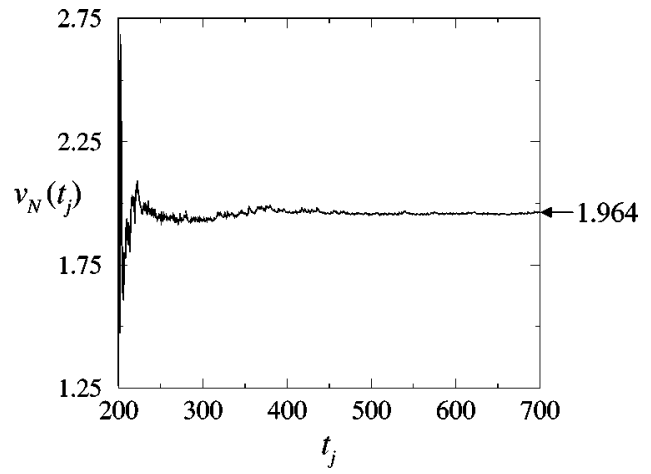

FIG. 4. Values of $v_{N}\left(t_{j}\right)$ for $200<t_{j}<700$ and $j$ $=1,2, \ldots, 980$. As expected, the fluctuations in $v_{N}\left(t_{j}\right)$ die out for large values of $j$.

therefore, allow us to set the error bar on the measurement of $v_{N}$ in Eq. (5.8), and we obtain

$$
v_{N}=1.964 \pm 0.006 \text {. }
$$

\section{B. The coefficient $A$ as a reflection of the nonlinear front behavior}

The quantity $A$ has been introduced to solve for the linear difference-differential equation, Eq. (3.1). Its numerical value, however, cannot be determined from the linear equation, since any value of $A$ satisfies it. To determine the value of $A$, therefore, one needs to solve the full nonlinear difference-differential equation, Eq. (2.6), expressed in terms of the comoving coordinate, $\xi$. This is done, together with the associated values of the real and imaginary part of $z$, in Appendix B; for $\tilde{\gamma}=1$ and $N=10^{4}$, we find

$$
A=0.961 \pm 0.012 \text {. }
$$

\section{Numerical test of the predictions for consistency of front shape and speed}

Equipped with the value of $A$ and the simulation data, we are now in a position to contrast the result of Sec. III with the simulation results. The purpose of this subsection is twofold: first, we demonstrate that the theoretical shape of the front generated in Sec. V B for $\tilde{\gamma}=1$ and $N=10^{4}$ agrees very well with the shape of the front obtained from the simulation data by taking snapshots at arbitrary times. Second, we demonstrate that there are significant differences in the two values of $v^{*}-v_{N}$, one obtained from Eq. (3.6), and the other from Eq. (5.8)

To compare the shapes of the front obtained from the theory and the simulations, we choose to take snapshots at three discrete times, $t=200, t=280$, and $t=360$. In Fig. 5 we present the plot that contains the front shapes for these times obtained from the simulations and the theory. The comoving coordinate $\xi$ for the bins is chosen in a way such that it coincides with the laboratory frame position of the front at $t=200$. As we can see from the graph below, the collapse of the data for $t=200, t=280$, and $t=360$ is very good and the solid line representing the theoretical prediction is almost

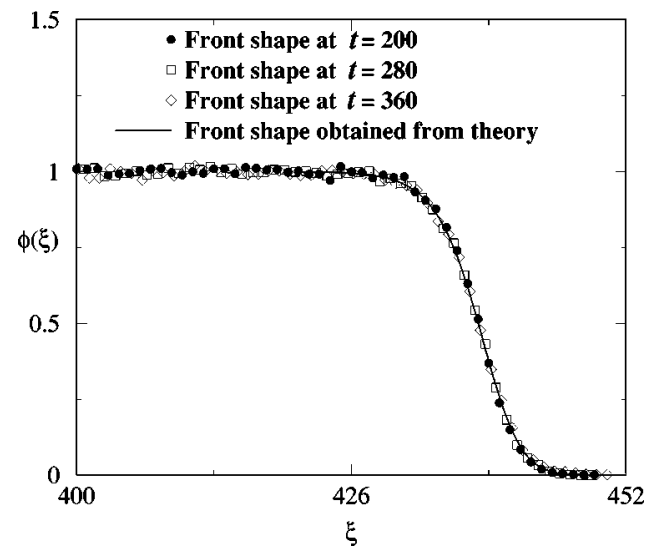

FIG. 5. Theoretical shape of the front represented by the solid line and the shape of the front obtained from computer simulations at three discrete times, $t=200, t=280$, and $t=360$, represented by three different symbols.

indistinguishable from the simulation data. The theoretical curve is first generated using the method described in Sec. $\mathrm{V}$ B with $v_{N}\left(t_{980}\right)$ as the asymptotic front speed, the corresponding values of $z_{l}, z_{r}, z_{i}$ and $A$ of Eqs. (B6) and (5.9), and then having it shifted to coincide with the laboratory frame position of the front at $t=200$.

We now return to the result of Sec. III, and denote $v^{*}$ $-v_{N}$ obtained from Eq. (3.6) by $\Delta v_{\text {asymp }}$. For $\tilde{\gamma}=1$, Eq. (3.2) yields $z_{0}=0.9071032 \ldots$ and $v^{*}=2.07344$. Using these values for $N=10^{4}$, we obtain

$$
\Delta v_{\text {asymp }}=0.152024 \ldots
$$

On the other hand, using $v_{N}\left(t_{980}\right)$ for $v_{N}$, the value $\Delta v_{\text {sim }}$ $=v^{*}-v_{N}$ comes out to be

$$
\Delta v_{\text {sim }} \equiv v^{*}-v_{N}\left(t_{980}\right) \approx 0.11
$$

which implies that the asymptotic estimate $\Delta v_{\text {asymp }}$ is about $38 \%$ larger than $\Delta v_{\text {sym }}$ from the computer simulations.

These results clearly indicate that for large but not extremely large values of $N\left(N=10^{4}\right.$ here $)$, there is much more to the story than $v^{*}-v_{N}$ being simply $\propto \ln ^{-2} N$. The theory presented in Sec. III does capture the essentials, and it would have been good enough to generate appropriate numbers for $v^{*}-v_{N}$, if one could obtain the value of $a$ externally. However, in view of the fact that the uniformly translating solution of Eq. (2.6) cannot be extended all the way up to the foremost bin, the quantity $a$ is fictitious and simply an effective quantity (already mentioned in Sec. III). Therefore, it is not possible to obtain the numerical value of $a$ from computer simulation results or from any theoretical estimate. Besides, the theory of Sec. III completely overlooks the microscopic intricacies at the tip of the front, and hence, it should be regarded as an effective theory.

\section{Numerical test of the theory for $\mathcal{P}(\Delta t)$}

In this subsection, we seek the numerical test of our theory presented in Sec. IV. We carry out this task in two steps. In the first step, we check most of the aspects of the 
theory for $N=10^{4}$ and $\tilde{\gamma}=1$, where we describe the method for obtaining $\mathcal{P}(\Delta t)$. Subsequently, in the second step, we check the predictions of our theory for $N=10^{2}, 10^{3}$, and $10^{5}$, keeping the value of $\tilde{\gamma}$ fixed at 1 . Notice that comparing probability distributions allows us to verify more detailed representations of the actual forward movements of the foremost bin against comparing only the asymptotic front speed $v_{N}$, which is the inverse of the first moment of $\mathcal{P}(\Delta t)$ [see Eq. (4.4)]. We should note that in view of the strong nonlinearity of the self-consistent theory of Sec. IV, we will have to use the values of $A, z_{r}, v_{N}$, and $z_{i}$ obtained from computer simulations to generate the $\mathcal{P}(\Delta t)$ curve, and then obtain the theoretical value of $v_{N}$. This process therefore becomes a self-consistency check of our theory of Sec. IV, as opposed to a verification of its predictions. Moreover, we do not compare the $\mathcal{P}(\Delta t)$ curves directly. This is for a very simple reason: namely, that the expression for $\mathcal{P}(\Delta t)$ in Eq. (4.7) involves $\left\langle N_{k_{m}}(\Delta t)\right\rangle$ as a coefficient. In an actual computer simulation, this quantity fluctuates wildly, and hence, generating a histogram to obtain the probability distribution $\mathcal{P}(\Delta t)$ from computer simulations proves to be difficult. Instead, we compare the "cumulative probability distribution" curves $P(\Delta t)$, which is defined as the probability of a new foremost bin creation happening at time $t \geqslant \Delta t$. From a theoretical point of view, the expression of $P(\Delta t)$ can be found easily from Eq. (4.7) as

$$
P(\Delta t)=\int_{\Delta t}^{\infty} d t^{\prime} \mathcal{P}\left(t^{\prime}\right)=\exp \left[-\tilde{\gamma} \int_{0}^{\Delta t} d t\left\langle N_{k_{m}}(t)\right\rangle\right] .
$$

It turns out that the $P(\Delta t)$ histogram generated from the computer simulation results is not noticeably affected by fluctuations, which makes its comparison with the $P(\Delta t)$ curve generated from our theory much simpler.

\section{The case of $\tilde{\gamma}=1$ and $N=10^{4}$}

The $P(\Delta t)$ curve from the computer simulations are generated in the following way: by definition $P(0)=1$. For $N$ $=10^{4}$ and $\tilde{\gamma}=1$, there are 980 values of $\Delta t$. First, these are arranged in an increasing order of magnitude, $\Delta t_{1}, \Delta t_{2}, \ldots, \Delta t_{980}$, and then in the corresponding values of $P(\Delta t)$ are obtained as

$$
P\left(\Delta t_{j+1}\right)=P\left(\Delta t_{j}\right)-\frac{1}{980}
$$

for $j=2,3, \ldots, 980$ with the initial condition that $P\left(\Delta t_{1}\right)$ $=1-1 / 980$. The corresponding $P(\Delta t)$ vs $\Delta t$ plot is shown in Fig. 6 by open circles.

To generate the corresponding theoretical cumulative probability distribution, we proceed in the following way. In a coordinate system, where the function $\phi_{k}^{(0)}(t=0)=0$ at $k$ $=\pi / z_{i}$ [which allows us to use the values of $A, z_{r}, v_{N}$, and $z_{i}$ of Eq. (B6)], we work out the whole machinery described by Eqs. (4.5)-(4.14) neglecting the fluctuation effects described in Sec. IV C. This process requires the value of $k_{m}$ $-k_{b}$, i.e., the number of bins at the tip of the front where the

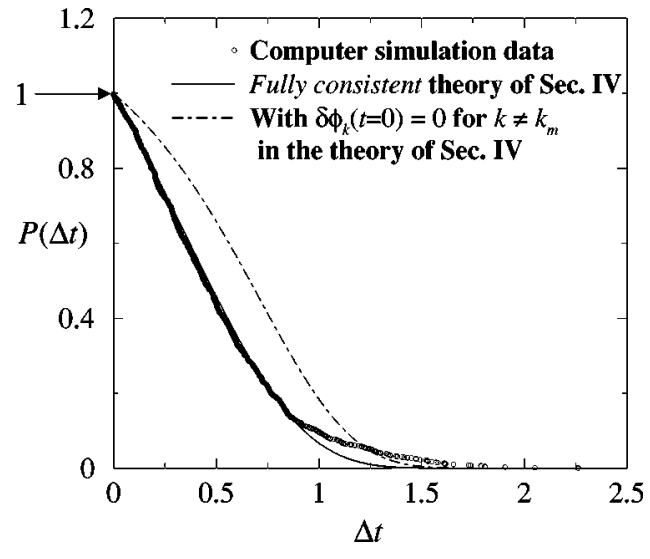

FIG. 6. The cumulative probability distribution $P(\Delta t)$ as a function of $\Delta t$ for $N=10^{4}$ and $\tilde{\gamma}=1$.

buildup of particle density is significant, as well as the value of $k_{m}-k_{m_{0}}$ as external inputs, and we choose $k_{m}-k_{b}=4$ for this purpose [38] (we refer back to Table I for the definitions of $k_{m_{0}}$, etc.). The calculation of the value of $k_{m}-k_{m_{0}}$ and the generation of the $\mathcal{P}(\Delta t)$ curve are carried out selfconsistently and hence by iteration, using the recursive feedback method [33]. However, to generate the $\mathcal{P}(\Delta t)$ curve for any guess value of $k_{m}-k_{m_{0}}$, one still needs to have the values of $\delta \Phi(t=0)$ for the $k_{m}-k_{b}$ bins at the tip as a starting point [see Eq. (4.12)]. At the same time, we notice that $\delta \Phi(t=0)$ can only be determined once the probability distribution $\mathcal{P}(\Delta t)$ is obtained. We choose to address this problem the following way: for any guess value of $k_{m}-k_{m_{0}}$, we start with Eq. (4.13) and $\delta \phi_{k}(t=0)=0$ for the rest of the $k_{m}-k_{b}$ bins. Keeping $k_{m}-k_{m_{0}}$ fixed, we then generate the corresponding $\mathcal{P}(\Delta t)$ curve and obtain the $\delta \phi_{k}(t=0)$ values for the rest of the $k_{m}-k_{b}$ bins using Eq. (4.14). We keep repeating this process until we converge in terms of the $\delta \phi_{k}(t=0)$ values, i.e., when the recursive correction to the values of $\delta \phi_{k}(t=0)$ becomes less than $10 \%$ of the $\delta \phi_{k}(t$ $=0)$ values at the previous step in the recursion. Once this point is reached for a value of $k_{m}-k_{m_{0}}$, we then compare the theoretical cumulative probability distribution $P(\Delta t)$ with Fig. 6 above to decide upon the next guess value of $k_{m}$ $-k_{m_{0}}$ in the recursive feedback method.

For $N=10^{4}$ and $\tilde{\gamma}=1$, the value of $k_{m}-k_{m_{0}}$ turns out to be $k_{m}-k_{m_{0}}=1.1431$. We present the corresponding theoretical cumulative probability curves in Fig. 6 . The solid line in Fig. 6 represents the fully consistent solution of Eqs. (4.5)(4.14), while the dashed line represents the theoretical cumulative probability curve obtained by solving Eqs. (4.5)(4.14) with $\delta \phi_{k}(t=0)=0$ for $k \neq k_{m}$. The fact that the fully consistent solution curve matches the computer simulation one much better than the naive approximation where all the $\delta \phi_{k}$ corrections behind the foremost bin are ignored is a strong indication of how significantly the buildup of particle densities in the bins behind the foremost one affects the property of $P(\Delta t)$. 


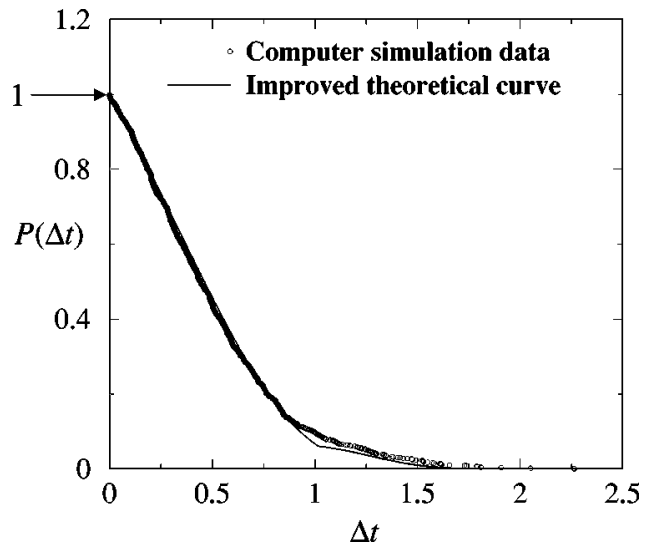

FIG. 7. The theoretical curve, which includes the effect of the vacant foremost bin events, and the simulation data for the cumulative probability distribution $P(\Delta t)$ are presented above for $N$ $=10^{4}$ and $\tilde{\gamma}=1$.

An examination of Fig. 6 immediately reveals that the agreement between the $P(\Delta t)$ curve generated by the fully consistent theory of Sec. IV and the computer simulation is extremely accurate up to about $\Delta t=0.9$. However, the theory is unable to capture the "tail" of the $P(\Delta t)$ curve for large $\Delta t$. Analysis of our data shows that this is due to the fluctuation effects discussed in Sec. IV C. As mentioned there, correlated diffusion events are not captured in this theory. However, we can follow the argument of Sec. IV C 2 to take into account the effect of the vacant foremost bin events on the $P(\Delta t)$ curve for large $\Delta t$ values: we assume that all cases of $\Delta t>t_{0} \approx 2 / v_{N}$ are due to the vacant foremost bin events. This means that for $\Delta t<t_{0}$, the $P(\Delta t)$ curve is given by the solid line in Fig. 6, but from $t_{0}$ onwards, the $P(\Delta t)$ curve must be generated from the mean-field dynamics of the tip described in Sec. IV, with the same value of $k_{m}$, but with the initial condition $\left\langle N_{k_{m}}\left(t=t_{0}\right)\right\rangle=N_{k_{m}}\left(t=t_{0}\right)=1 / N$, as opposed to $\left\langle N_{k_{m}}(t=0)\right\rangle=N_{k_{m}}(t=0)=1 / N$. With the value of $k_{m}$ already determined in this subsection, the corresponding equations, Eqs. (4.5)-(4.14), are easy to solve selfconsistently as before. From this analysis, we obtain the behavior of $P(\Delta t)$ for $\Delta t>t_{0}$, having noticed that $P\left(\Delta t \rightarrow t_{0}\right.$ + ) must be the same as the value obtained from the solid line in Fig. 6 at $\Delta t=t_{0}$, i.e., 0.06034 . We present the final theoretical $P(\Delta t)$ curve together with the simulation data in Fig. 7. Notice that this process introduces a finite discontinuity in the density of the $X$ particles inside the foremost bin at $\Delta t=t_{0}$, since for $\Delta t<t_{0}$, the density of the $X$ particles in the foremost bin is obtained from a fully consistent theory of

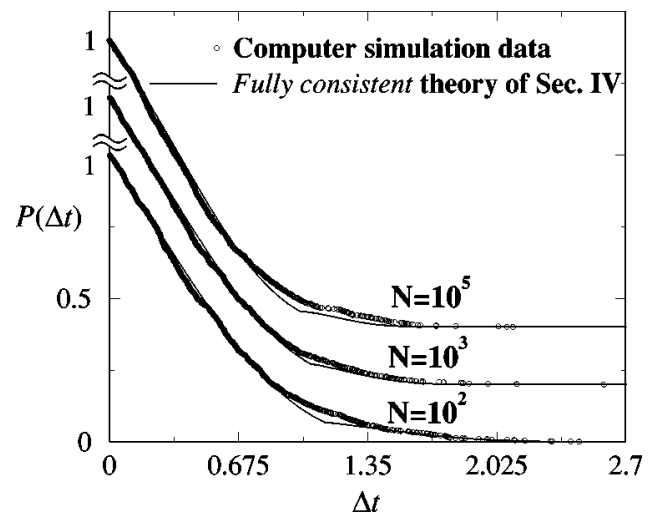

FIG. 8. The combined theoretical curve and the simulation data for the cumulative probability distributions $P(\Delta t)$ vs $\Delta t$ for $N$ $=10^{2}, 10^{3}$, and $10^{5}$, and $\tilde{\gamma}=1$. The curves for the latter two are shifted upwards for clarity.

Sec. IV, while at $\Delta t=t_{0}$, it is set equal to $1 / N$ manually. As a consequence, the theoretical $P(\Delta t)$ curve in Fig. 7 has a slope discontinuity at $\Delta t=t_{0}$.

In Fig. 7, the improved theoretical curve follows the curve reasonably well, but it still lies below the simulation data points for $\Delta t \geqq 2 / v_{N}$, as it should be. This discrepancy gives us an idea about the effect of the correlated diffusion events on the $P(\Delta t)$ curve that we could not estimate. Using Eq. (5.7) to calculate the front speed from the theoretical curve in Fig. 7, we obtain

$$
v_{N}(\text { theoretical })=1.98882 \text {. }
$$

This is about 0.024 higher than the asymptotic front speed measured by the computer simulation [see Eq. (5.8)], in agreement with the fact that the theoretical curve for $P(\Delta t)$ slightly underestimates the simulation one for $\Delta t \gtrsim 2 / v_{N}$.

\section{The cases of $N=10^{2}, 10^{3}$, and $10^{5}$, with $\tilde{\gamma}=1$}

We now further test our theory for $N=10^{2}, 10^{3}$, and $10^{5}$, keeping the value of $\tilde{\gamma}$ fixed at 1 . The values of $v_{N}$ (simulation), $z_{r}, z_{i}$, and $A$ in Table II below. The corresponding $P(\Delta t)$ vs $\Delta t$ graphs, which are the analogs of the graph in Fig. 7, have been plotted together in Fig. 8. Table II presents the theoretical values of $v_{N}$ that are calculated using these $P(\Delta t)$ vs $\Delta t$ graphs, and predicted $v_{N}$ from Eq. (3.6).

Notice that as $N$ decreases, according to Table II, the value of $z_{r}$ decreases more and more from its $N \rightarrow \infty$ limit $z_{0}$, while $z_{i}$ increases. This is an illustration of how the nonmean-field effects become increasingly important behind the

TABLE II. The $v_{N}$ (simulation), $z_{r}, z_{i}, A, v_{N}$ (theoretical), and $v_{N}$ [Eq. (3.6)] values for $\tilde{\gamma}=1$, and $N$ $=10^{2}, 10^{3}$, and $10^{5}$. The $v_{N}$ (theoretical) values are calculated from the theoretical curves of Fig. 8 .

\begin{tabular}{lcccccc}
\hline \hline$N$ & $v_{N}$ (simulation) & $z_{r}$ & $z_{i}$ & $A$ & $v_{N}$ (theoretical) & $v_{N}$ [Eq. (3.6)] \\
\hline $10^{2}$ & 1.778 & 0.8217 & 0.436 & 0.8836 & 1.808 & 1.465 \\
$10^{3}$ & 1.901 & 0.8586 & 0.3313 & 0.9042 & 1.899 & 1.803 \\
$10^{5}$ & 2.001 & 0.8885 & 0.2654 & 1.0714 & 2.057 & 1.976 \\
\hline \hline
\end{tabular}



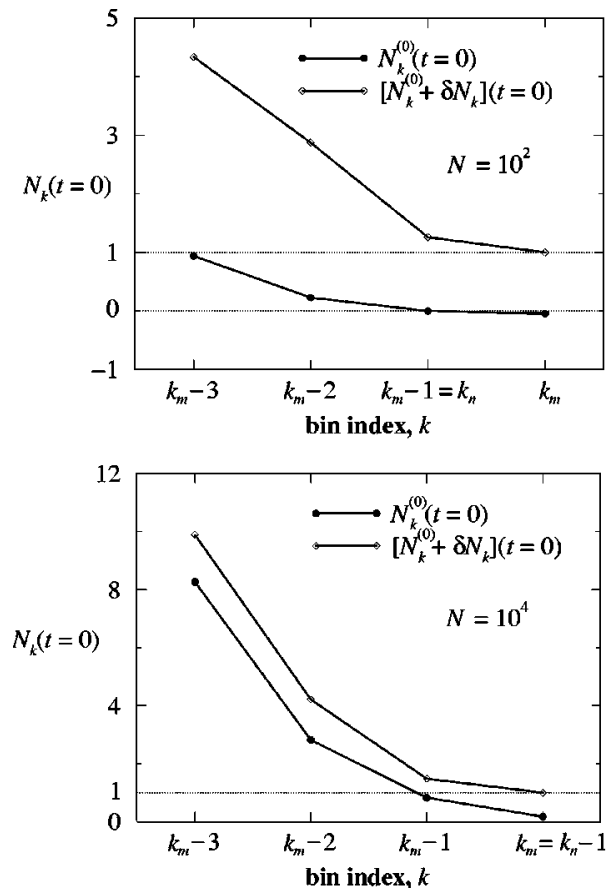
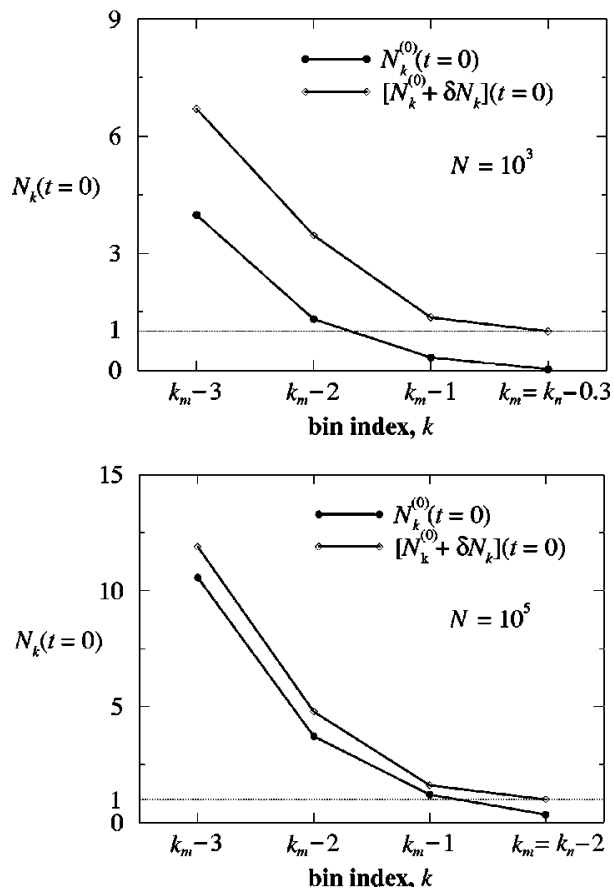

FIG. 9. Comparison between the $\left\langle\delta N_{k}\right\rangle(t=0)$ and the $\left\langle N_{k}^{(0)}\right\rangle(t$ $=0)$ values for the four foremost bins and for $N=10^{2}, 10^{3}, 10^{4}$, and $10^{5}$. The angular brackets for the notations have been omitted in the figure for clarity. Note that as $N$ increases, the corrections $\left\langle\delta N_{k}\right\rangle(t=0)$ compared to the $\left\langle N_{k}^{(0)}\right\rangle(t=0)$ profile become less and less important. tip region. We should also note two more points about Fig. 8: (i) in the absence of any estimate of the correlated diffusion events for $\Delta t \gtrsim 2 / v_{N}$, the theoretical curves lie below the simulation data (although for $N=10^{2}$ and $10^{3}$, it is not so clearly discernible), and (ii) the agreement between the theoretical $P(\Delta t)$ curve and the simulation one for $N=10^{5}$ may appear to be worse than the corresponding ones for $N$ $=10^{2}, 10^{3}$, and $10^{4}$, but this may be due to the fact that we have had to continuously cut off particles from the saturation region of the front on the left to obtain the stochastic simulation data for $N=10^{5}$ within a reasonable computer time. We have found that the shape of the $P(\Delta t)$ histogram obtained from the simulation gets slightly modified depending on how this subtraction in carried out, specially in the $\Delta t$ $\gtrsim 2 / v_{N}$ region.

We now return to [the issue raised in point (5) of Sec. IV D] the increased importance of the stalling effects and the deviations from the $N \rightarrow \infty$ asymptotic theory for decreasing values of $N$. Figure 9 shows the comparison between the actual particle numbers, $\left\langle\delta N_{k}\right\rangle(t=0)=N \delta \phi_{k}(t=0)$ and the $\left\langle N_{k}^{(0)}\right\rangle(t=0)=N \phi_{k}^{(0)}(t=0)$ values for four foremost bins, i.e., for $k=k_{m}, k_{m-1}, k_{m-2}$, and $k_{m-3}$ (note that for the sake of clarity, we have omitted the angular brackets for notations in Fig. 9). These values have been obtained selfconsistently, while generating the theoretical curves of Figs. 7 and 8. As expected, it is clear that the $\delta N_{k}(t=0)$ values are playing less and less role for increasing value of $N$. There are couple of more points that one must take notice of. First, as can be seen from Table II, $A$ increases with $N$, but only by a small amount. Second, it is also clear from Fig. 9 that with respect to the $k_{n}$ th bin [where $\left\langle N^{(0)}\right\rangle$ vanishes], the position of the $k_{m}$ th bin (where $\left\langle N^{(0)}+\delta N\right\rangle(t=0)=1$ ) shifts gradually towards the left for increasing $N$ (see Table I for the definition of $k_{n}$ ). All these together elucidate that for not very large values of $N$, the actual $N$ dependence of the front speed $v_{N}$ is a much more complicated story than simply the $1 / \ln ^{2} N$ relaxation to $v^{*}$ of $v_{N}$. From the trend of the gradual left shifting of $k_{m}$ (with respect to $k_{n}$ ) and the gradual unimportance of the role of $\delta N_{k}(t=0)$ values compared to their $N_{k}(t=0)$, it is conceivable that for extremely large values of $N, k_{m} \rightarrow k_{m_{0}}$, and $\delta N_{k}(t=0) \rightarrow 0$, and it is this limit where the cutoff (at $\left.\phi^{(0)}=1 / N\right)$ picture in Ref. [9] becomes applicable. In this sense, the theory of Sec. IV is complementary to that of Ref. [9], as together they span the whole range of $N$ values, from reasonably small to very large.

\section{E. The case of $\tilde{\gamma}=0.1$ and $N=10^{4}$}

We now investigate the claim made in Sec. IV that the correlated diffusion events affect the $\mathcal{P}(\Delta t)$ curve so severely for low diffusion coefficients that our approach fails badly, by comparing the theoretical $P(\Delta t)$ curve with the simulation one, for $\tilde{\gamma}=0.1$ and $N=10^{4}$.

We present the two curves in Fig. 10. The asymptotic speed for the corresponding pulled front, $v^{*}$, for this set of parameter values is given by $v^{*}=0.7754$ and the simulation results yield $v_{N}=0.698$.

The theoretical curve of Fig. 10 is analogous to that of Fig. 6 represented by the solid line, and it is obtained by means of a fully consistent theory of Sec. IV. Notice that the agreement between the theory and the computer simulation results is not good beyond $\Delta t \approx 1 / v_{N}$. It is also obvious that an attempt to incorporate the effect of the vacant foremost bin events does not do any improvements in this case, since the value of $P(\Delta t)$ is almost zero for $\Delta t \gtrsim 2 / v_{N}$. This is very much expected and a careful examination of the simulation data also reveals that the vacant foremost events do not occur at all during the front speed measurement times between $t$ 


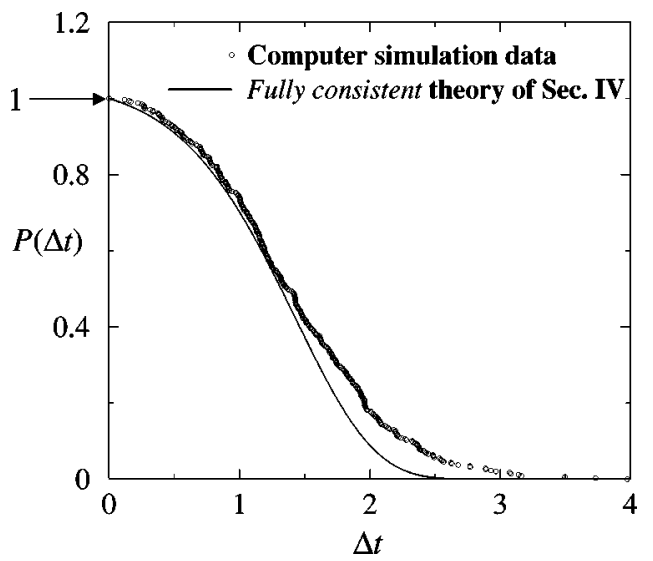

FIG. 10. The cumulative probability distribution $P(\Delta t)$ as a function of $\Delta t$ for $N=10^{4}$ and $\tilde{\gamma}=0.1$.

$=200$ and $t=700$ [see Eq. (5.6) and the paragraph above it]. Altogether, this fits very nicely in the consistent picture that we have put forward so far, which simply indicates that the entire discrepancy between the theory and the computer simulation in Fig. 10 is solely due to the correlated diffusion events.

\section{THE CASE OF A FINITE NUMBER OF Y PARTICLES ON THE LATTICE SITES}

We now briefly turn our attention to the following reaction-diffusion process $X+Y \rightarrow 2 X$ on a lattice: at each lattice position, there exists a bin. Once again, we label the bins by their serial indices $k, k=1,2,3, \ldots, M$, placed from left to right. In the $k$ th bin, there are a certain number of $X$ particles, denoted by $N_{X, k}$ and a certain number of $Y$ particles, denoted by $N_{Y, k}$. Both $N_{X, k}$ and $N_{Y, k}$ are finite. The dynamics of the system is described by three basic processes:

(i) Diffusion of the $X$ particles in the $k$ th bin to the ( $k$ $-1)$ th or the $(k+1)$ th bin with a rate of diffusion $\tilde{\gamma}$. If an $X$ particle in bin 1 jumps towards the left, or an $X$ particle in the $M$ th bin jumps to the right, then they are immediately replaced.

(ii) Likewise, diffusion of the $Y$ particles in the $k$ th bin to the $(k-1)$ th or the $(k+1)$ th bin with a rate of diffusion $\tilde{\gamma}$. If an $X$ particle in bin 1 jumps towards the left, or an $X$ particle in the $M$ th bin jumps to the right, then they are immediately replaced.

(iii) Reaction to produce an extra $X$ particle having annihilated a $Y$ particle $(X+Y \rightarrow 2 X)$, with a rate $1 / N$.

To study the phenomenon of front propagation for this model, the initial configuration of the system is taken as $N_{X, k}=N\left[1-\Theta\left(k-k_{0}\right)\right]$ and $N_{Y, k}=N \Theta\left(k-k_{0}\right)$ (a step function in the density of the $X$ particles). Because of the reaction process (iii), the number of $X$ particles in any bin keeps increasing, until the supply of $Y$ particles in that bin runs out. As a result, the region that is full of $X$ particles slowly invades the region that is full of $Y$ particles, and this constitutes a propagating front.

The corresponding equation of the front that is analogous

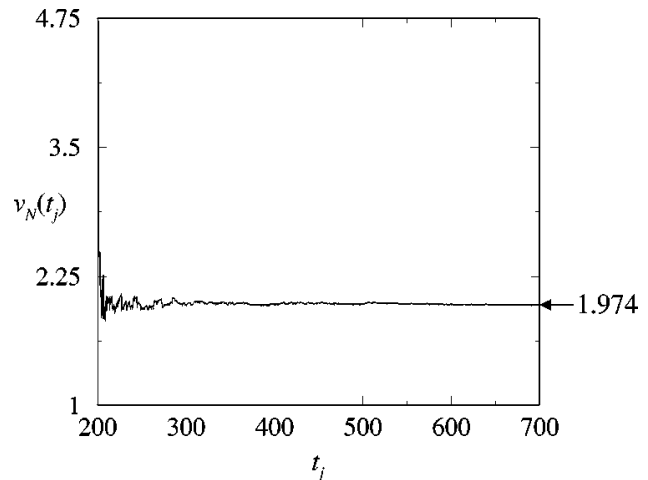

FIG. 11. Values of $v_{N}\left(t_{j}\right)$ for $200<t_{j}<700$ and $j$ $=1,2, \ldots, 980$. As expected, the fluctuations in $v_{N}\left(t_{j}\right)$ die out for large values of $j$.

to Eq. (2.5), is slightly more complicated, and it is given by

$$
\begin{aligned}
\frac{\partial}{\partial t}\left\langle N_{X, k}(t)\right\rangle= & \tilde{\gamma}\left[\left\langle N_{X, k+1}(t)\right\rangle+\left\langle N_{X, k-1}(t)\right\rangle-2\left\langle N_{X, k}(t)\right\rangle\right] \\
& +\frac{1}{N}\left[\left\langle N_{k}(t) N_{X, k}(t)\right\rangle-\left\langle N_{X, k}^{2}(t)\right\rangle\right]
\end{aligned}
$$

where $N_{k}(t)$ is the total number of particles in the $k$ th bin at time $t$. In Eq. (6.1), if we replace $\left\langle N_{k}(t) N_{X, k}(t)\right\rangle$ by $\left\langle N_{k}(t)\right\rangle\left\langle N_{X, k}(t)\right\rangle=N\left\langle N_{X, k}(t)\right\rangle$, then one retrieves Eq. (2.5). In this section, therefore, our purpose is to investigate if the correlation term $\left\langle N_{k}(t) N_{X, k}(t)\right\rangle$ has any bearing on the corrections of the asymptotic front speed over its corresponding value obtained from the model analyzed so far.

Front propagation in this model has been studied numerically by Kessler and coauthors [10]. Our interest in this model is motivated by the following observation: in terms of the average number of $X$ particles in a bin, an appropriate reaction rate yields an equation, which is similar to Eq. (2.5). However, the linear growth term of Eq. (2.5) is replaced by a more complicated correlation function between the number of $X$ and $Y$ particles in the $k$ th bin. Nevertheless, near the foremost bin of the $X$ particles, the number of $Y$ particles is so large that the fluctuations in their number remains small. Upon neglecting these fluctuations, the linear growth term for the $X$ particles becomes the same as the one before, and one therefore expects the speed correction to stay unaffected. Our purpose is to check this expectation numerically, by comparing data for the front speed in this model with those given in Eq. (5.8) for Eq. (2.5). The algorithm that we use in our simulation is similar to that of Sec. V A. The value of $M$ is taken to be 1500 and for the starting configuration of the system, we use $k_{0}=50$.

The asymptotic front speed is calculated using Eqs. (5.6) and (5.7). The measurement of the front speed starts at $t$ $=200$ and stops at $t=700$. There are $985 \Delta t$ values in this time interval. The corresponding $v_{N}\left(t_{j}\right)$ vs $t_{j}$ graph is shown below in Fig. 11. Using the same method of calculation as in Sec. V A, the asymptotic front speed for $N=10^{4}$ and $\tilde{\gamma}=1$ comes out of the computer simulation as 


$$
v_{N}=1.974 \pm 0.009 .
$$

We notice that the error bars of Eqs. (5.8) and (6.2) overlap with each other, and we conclude that the correlations between the total number of particles and the number of $X$ particles in the bins [the $\left\langle N_{k}(t) N_{X, k}(t)\right\rangle$ term in Eq. (6.1)] indeed do not affect the asymptotic front speed, as we had expected.

As noted before, Kessler et al. [10] claimed that the prefactor of the speed correction was about a factor 2 different from the value expected from the asymptotic formula (3.6) of Brunet and Derrida. Whether this is due to the values of $N$ not being large enough, or due to their particular way of implementing the stochastic simulations, is unclear to us. From our data, there is no reason to believe that for large $N$ there is an essential difference between the model with finite number of $Y$ particles and the earlier model with an infinite supply of $Y$ particles, otherwise the asymptotic formula would be incorrect as $N \rightarrow \infty$.

\section{CONCLUSION AND OUTLOOK}

In this paper we have identified a large number of effects that play a role in the tip region of fluctuating fronts which in the mean-field limit reduce to pulled fronts. While a full theory from first principles, which yields explicit predictions for the front speed for finite $N$ will be hard to come by, we believe that any such theory will incorporate most of the effects we have analyzed and studied with computer simulations. One important conclusion from our studies is that while the asymptotic large- $N$ correction derived by Brunet and Derrida is universal (in the sense of being independent of the details of the model) the corrections to this expression do depend on many details of the model. In most cases, deviations from the asymptotic results are significant for values of $N$ that can realistically be studied.

The message of this paper is as follows: the bulk of a fluctuating front can still be considered a uniformly translating one and one can properly define a comoving coordinate system, in which the shape of the bulk remains unchanged; on the other hand, the position of the tip of the front in such a comoving coordinate system fluctuates, and only on average the tip becomes stationary in this comoving coordinate system. From the mean-field limit of this fluctuating front, we know that the tip region is very important for its dynamics; as a result, the fluctuating tip plays a very significant role in deciding the asymptotic front speed, in which two very important aspects come to play a role-discrete nature of particles and discrete nature of the bin indices. In this paper, we have tried to formulate a theory to model this fluctuating tip. This theory is still a mean-field type theory. More specifically, at $t=0$, the shape of the tip is always the same mean shape, and hence this theory is unable to capture the correlated diffusion events or the vacant foremost bin events (although we can estimate the effect of the latter). Any alternative theory, that one might think at this point, must be able to take into account these fluctuation effects, which, as previously explained, must be able to study multitime correlation functions for correlated jumps at the tip.
The prospect of such a theory, however, looks grim at this point. Not only the problem becomes highly nonlinear, but also one must realize that the fluctuations in the number of $X$ particles in the bins near the tip is of the same order as the number of $X$ particles in them $(\sim 1)$, and there does not exist any small parameter that one can do perturbation theory with.

Finally, we note here that we have confined our analysis to cases in which the growth and hopping terms for few particles per site or bin are the same as those for a finite density of particles. In such cases the front speed converges to $v^{*}$ as $N \rightarrow \infty$. One should keep in mind, though, that there are also cases where with minor modifications of the stochastic rules for few particles, one can arrive at a situation in which the speed does not converge to the pulled speed $v^{*}$ as $N \rightarrow \infty$, even though in the mean-field limit one obtains a dynamical equation that admits pulled fronts. We have discussed this in more detail elsewhere [27].

\section{ACKNOWLEDGMENTS}

D.P. wishes to acknowledge many important, motivating, and stimulating discussions with Hans van Leeuwen, Ramses van Zon, and Zohar Nussinov, the help from Ellak Somfai on setting up the computer simulations used to obtain results in this paper, and the financial support from "Fundamenteel Onderzoek der Materie" (FOM).

\section{APPENDIX A: SUMMARY OF THE DERIVATION OF THE GENERALIZED VELOCITY CORRECTION FORMULA}

In this appendix, we derive the generalized velocity correction formula, Eq. (3.4), and its interpretation. Without any loss of generality, we can express the front solution $\phi(\xi)$ for $v_{N}<v^{*}$ by (cf. Refs. [9-11,16,17])

$$
\phi(\xi)=A \sin \left[z_{i} \xi+\beta\right] \exp \left(-z_{r} \xi\right)
$$

at the leading edge of the front, where $z_{r}=\operatorname{Re}(z)$ and $z_{i}$ $=\operatorname{Im}(z)$. The corresponding dispersion relation is then given by

$$
\begin{gathered}
z_{r} v_{N}=2 \tilde{\gamma}\left(\cosh z_{r} \cos z_{i}-1\right)+1 \text { and } \\
z_{i} v_{N}=2 \tilde{\gamma} \sinh z_{r} \sin z_{i} .
\end{gathered}
$$

The additive phase $\beta$ in Eq. (3.3) can be scaled away by redefining $A$ and the position of the origin from where $\xi$ is measured. We therefore drop $\beta$ in this appendix. Since the scaled particle density has to be positive, i.e., $\phi(\xi) \geqslant 0$, the physical linear solution regime must be confined within the range where $0<z_{i} \xi \leqslant \pi$. We now make a notational choice to denote the comoving coordinate corresponding to the node of the sine function in Eq. (3.3), by $\xi_{c}+1$, i.e.,

$$
z_{i}\left(\xi_{c}+1\right)=\pi
$$

One should understand at this point that although Eq. (A1) suggests that there is a second node of $\phi(\xi)$, where the argument of the sin function becomes zero, such a node does 
not exists. Much before the argument of the $\sin$ function becomes zero, the nonlinear saturation term becomes important and the solution (A1) for the linearized equation does not hold any longer. In this overly simplified mean-field description, $\xi_{c}$ plays the role of the comoving coordinate of the foremost bin. The mean-field description of the front is then completed by claiming that $\phi(\xi)=0$ for $\xi \geqslant \xi_{c}+1$. Let us also denote the density of the $X$ particles in the "foremost bin," which in this approximation is at $\xi_{c}$, by $a / N$, to have

$$
A \sin \left[z_{i} \xi_{c}\right] \exp \left(-z_{r} \xi_{c}\right)=\frac{a}{N} .
$$

Once the parameters $A$ and $a$ are known, Eqs. (A2)-(A4) form a set of four equations for four unknowns, $z_{r}, z_{i}, \xi_{c}$, and $v_{N}$, which we can then solve numerically for the asymptotic front speed $v_{N}$.

In order to put our results in a particular form that facilitates comparison with the earlier results in literature [9], we analyze Eqs. (A2)-(A4) for large $N$. First, with the help of the Eq. (A3), we reduce Eq. (A4) to

$$
A \sin z_{i} \exp \left(-z_{r} \xi_{c}\right)=\frac{a}{N} .
$$

Next, having introduced a new variable $\mu$, such that

$$
\xi_{c}=\frac{\ln N}{z_{r}}+\mu,
$$

and using Eq. (A3), Eq. (A5) is further reduced to an implicit equation in $\mu$,

$$
z_{r} \mu=\left[\ln \frac{A}{a}+\ln \left\{\sin \frac{\pi z_{r}}{\ln N+1+\mu}\right\} .\right.
$$

Since $N$ is large, one can solve this implicit equation $\mu$ by means of a simple successive approximation procedure. At the lowest order, one can drop the $\mu$ term in the denominator of the argument of the sine function in Eq. (A7) and obtain

$$
\mu \approx \frac{1}{z_{r}}\left[\ln \frac{A}{a}+\ln \left\{\sin \frac{\pi z_{r}}{\ln N+1}\right\} .\right.
$$

Finally, $z_{i}$ can be obtained from Eqs. (A3), (A6), and (A8) as

$$
z_{i} \approx \frac{\pi z_{r}}{\ln N+z_{r}+\ln \frac{A}{a}+\ln \left[\sin \frac{\pi z_{r}}{\ln N+1}\right]}
$$

By now, we have eliminated the unknown $\xi_{c}$ and reduced the problem to solving three unknowns, $z_{r}, z_{i}$, and $v_{N}$ from three equations, Eqs. (A2) and (A9). From Eq. (A9), one can see that for large $N$, the approach of $z_{i}$ to zero is extremely slow, going only as $\ln ^{-1} N$ and also the fact that for the strict limit of infinite $N, z_{i}=0$, which reduces Eq. (A2) to Eq. (3.2), as it should. For large $N$, therefore, one expects that $z_{r} \approx z_{0}$ and $z_{i} \ll 1$, and one can expand $v_{N}$ around its value for $z=z_{0}$. Upon expanding $v_{N}$ around $v^{*}$, and using the solution of $z_{i}$ from Eq. (A9) with $z_{r}$ replaced by $z_{0}$, we then find that the asymptotic speed $v_{N}$ is given by Eq. (3.4).

\section{APPENDIX B: DETERMINATION OF $A$}

Solving the full nonlinear difference-differential equation, Eq. (2.6), is not an easy task. For a given set of values of $\tilde{\gamma}$ and $N$, there are essentially two methods to determine the value of $A$. The first one is to obtain the solution close to the saturation value $\phi=1$ and thereafter iterate the solution until one reaches $\phi \approx 0$. Close to the saturation value $\phi=1$, one can define $\eta(\xi)=1-\phi(\xi)$, which reduces Eq. (2.6) to an equation in $\eta(\xi)$, given by

$$
-v_{N} \frac{d \eta}{d \xi}=\tilde{\gamma}[\eta(\xi+1)+\eta(\xi-1)-2 \eta(\xi)]-\eta(\xi)+\eta^{2}(\xi) .
$$

For $\eta$ values close to zero, the solution of Eq. (5.7) is given by the linearized equation

$$
-v_{N} \frac{d \eta}{d \xi}=\tilde{\gamma}[\eta(\xi+1)+\eta(\xi-1)-2 \eta(\xi)]-\eta(\xi),
$$

with the corresponding solution $\eta(\xi)=B_{1} \exp \left[z_{l}\left(\xi-\xi_{0}\right)\right]$. Substitution of this solution in Eq. (B2) yields the dispersion relation between $v_{N}$ and $z_{l}$,

$$
-v_{N} z_{l}=2 \gamma\left[\cosh z_{l}-1\right]-1 .
$$

One can then iterate this solution towards $\phi=0$. The full solution of Eq. (B1) can be written as

$$
\eta(\xi)=\sum_{n=1}^{\infty} B_{n} \exp \left[n z_{l}\left(\xi-\xi_{0}\right)\right]
$$

where the corresponding $B_{n}$ values are obtained from the recursion relation

$$
B_{n}=\frac{B_{1} B_{n-1}+B_{2} B_{n-2}+\cdots+B_{n-1} B_{1}}{1-n v_{N} z_{l}-2 \tilde{\gamma}\left(\cosh n z_{l}-1\right)} .
$$

As a starting point for constructing the solution near $\phi=1$, one can choose arbitrary values of $\xi_{0}$ and $B_{1}$, so long as the value of $B_{1}$ is sufficiently small. At small values of $B_{1}$, any scaling of $B_{1}$ amounts to a simple shift of the origin $\xi_{0}$. Finally, one can then match the solution, thus obtained, to the form $\phi(\xi)=A \sin \left[z_{i}\left(\xi-\xi_{1}\right)\right] \exp \left[z_{r}\left(\xi-\xi_{1}\right)\right]$ near $\phi=0$ and determine the value of $A$.

The second method to obtain the numerical value of $A$ is to assume a certain value of $A$ close to $\phi=0$ with the functional form $\phi(\xi)=A \sin \left[z_{i}\left(\xi-\xi_{1}\right)\right] \exp \left[z_{r}\left(\xi-\xi_{1}\right)\right]$ and then continue to iterate the corresponding solution in the direction of $\phi=1$ in a similar manner. This time, if the assumed value of $A$ is correct, then close to $\phi=1$, one must recover the exponential behavior of $\phi(\xi)$, as in Eq. (B4). However, we have found that the first method is stable under small 
changes in the starting value of $B_{1}$, while the second method is not stable under small changes in the assumed value of $A$.

The first method should therefore be the natural choice, albeit from a practical point of view, one needs a very large number of $B_{n}$ values to extend the solution of $\phi(\xi)$ all the way up to $\phi=0$. In practice, we have therefore used a "double shooting" method [39], in which the functions are calculated from both sides, and then matched somewhere in the middle.

The matching of the values of the functions and their derivatives at $\phi_{0}$ requires the values of $z_{l}, z_{r}$, and $z_{i}$ to be determined externally. For $\tilde{\gamma}=1$ and $N=10^{4}$, the values of $z_{l}, z_{r}, z_{i}$, and $A$ are numerically obtained from Eqs. (5.8), (A2), and (B3) as

$$
\begin{gathered}
z_{l}=0.4187 \mp 0.0008, \\
z_{r}=0.877 \pm 0.002, \\
z_{i}=0.264 \mp 0.007, \\
A=0.961 \pm 0.012 .
\end{gathered}
$$

Of course, the numerical value of $A$ depends on the origin, where from $\xi$ is measured for the form in Eq. (3.3). In Eq. (B6) above, the value of $A$ is determined with $\beta=0$, i.e., the solution of the linearized equation at the leading edge of the front is zero at $\xi=\pi / z_{i}$. We mention here that the uncertainty in these numbers is determined by the uncertainty in $v_{N}$, not by the inaccuracy of the numerical method.
[1] E. Meron, Phys. Rep. 218, 1 (1992).

[2] G. Zocchi, P. Tabeling, and M. Ben Amar, Phys. Rev. Lett. 69, 601 (1992).

[3] Y. Tu and M.C. Cross, Phys. Rev. Lett. 69, 2515 (1992).

[4] U. Ebert, W. van Saarloos, and C. Caroli, Phys. Rev. E 55, 1530 (1997).

[5] M.C. Cross and P.C. Hohenberg, Rev. Mod. Phys. 65, 851 (1993).

[6] H.P. Breuer, W. Huber, and F. Petruccione, Physica D 73, 259 (1994).

[7] A. Lemarchand, A. Lesne, and M. Mareschal, Phys. Rev. E 51, 4457 (1995).

[8] J. Armero, J.M. Sancho, J. Casademunt, A.M. Lacasta, L. Ramŕez-Piscina, and F. Sagués, Phys. Rev. Lett. 76, 3045 (1996).

[9] E. Brunet and B. Derrida, Phys. Rev. E 56, 2597 (1997); Comput. Phys. Commun. 122, 376 (1999); J. Stat. Phys. 103, 269 (2001).

[10] D.A. Kessler, Z. Ner, and L.M. Sander, Phys. Rev. E 58, 107 (1998).

[11] L. Pechenik and H. Levine, Phys. Rev. E 59, 3893 (1999).

[12] J. Armero, J. Casademunt, L. Ramŕez-Piscina, and J.M. Sancho, Phys. Rev. E 58, 5494 (1998).

[13] J. Riordan, C.R. Doering, and D. ben-Avraham, Phys. Rev. Lett. 75, 565 (1995).

[14] G. Dee and J.S. Langer, Phys. Rev. Lett. 50, 383 (1983).

[15] E. Ben-Jacob, H.R. Brand, G. Dee, L. Kramer, and J.S. Langer, Physica D 14, 348 (1985).

[16] W. van Saarloos, Phys. Rev. A 39, 6367 (1989).

[17] U. Ebert and W. van Saarloos, Physica D 146, 1 (2000).

[18] A. Rocco, U. Ebert, and W. van Saarloos, Phys. Rev. E 62, R13 (2000).

[19] G. Tripathy and W. van Saarloos, Phys. Rev. Lett. 85, 3556 (2000)

[20] G. Tripathy, A. Rocco, J. Casademunt, and W. van Saarloos, Phys. Rev. Lett. 86, 5215 (2001).

[21] D. Panja and W. van Saarloos, Phys. Rev. E 66, 015206(R) (2002)

[22] M. Kardar, G. Parisi, and Y.C. Zhang, Phys. Rev. Lett. 56, 889 (1986).

[23] One should be careful in considering the limits in discussing this issue. Strictly speaking, for any finite particle number $\mathrm{N}$ a front is not pulled, but weakly pushed. Hence for any finite $N$ the true asymptotic scaling should be of the KPZ type.

[24] The issue is complicated, as there are two effects which may play a role: besides the crossover to KPZ behavior because the finite particle effects make the front weakly pushed, it has been questioned whether the multiplicative noise used in Refs. $[18,20]$ is consistent with intrinsic fluctuations, E. Moro, Phys. Rev. Lett. 87, 238303 (2001).

[25] U. Ebert and W. van Saarloos, Phys. Rep. 337, 139 (2000).

[26] R. van Zon, H. van Beijeren, and Ch. Dellago, Phys. Rev. Lett. 80, 2035 (1998).

[27] D. Panja and W. van Saarloos, Phys. Rev. E 65, 057202 (2002).

[28] R.A. Fisher, Ann. Eugenics 7, 355 (1937).

[29] A. Kolmogoroff, I. Petrovsky, and N. Piscounoff, Bull. Univ. Moscou, Ser. Int., Sec. A 1, 1 (1937).

[30] For the FKPP model with a cutoff in the growth term that Brunet and Derrida [9] introduced to model the finite particle effects, it is easy to convince oneself explicitly that the fronts are in fact always weakly pushed, according to the classification of Ref. [17].

[31] It is useful to realize that pulled fronts on a lattice without a finite particle cutoff are already somewhat exceptional, in that the lattice effects hardly play a role. Fronts between two linearly stable states are much more sensitive to lattice effects, e.g., they can become "locked" over some nonzero range of driving forces [see, e.g., G. Fáth, Physica D 116, 176 (1998)]. The lattice effects we analyze here are less dramatic, but of a similar nature.

[32] We note that several aspects of the analysis in this section show similarities with elements of the analysis of Ref. [9]. However, their analysis is simpler because they introduced a cutoff in a phenomenological way into the growth rate in the continuum mean field equation. As a result, they could simply match the two regions where the growth rate was different. Our analysis is beset by the fact that we do attempt to take the lattice effects into account (this already makes matching impossible), as well as the stalling effects and the changes this gives in the profile behind the foremost bin.

[33] This means that we start with a guess value of $k_{m}$, obtain the 
theoretical $\mathcal{P}(\Delta t)$ curve, compare it with the $\mathcal{P}(\Delta t)$ curve obtained from the computer simulations, and use the nature of discrepancy to make the next guess value of $k_{m}$. This process is repeated until one converges to the value of $k_{m}$, for which the best agreement between the theoretical and the simulation $\mathcal{P}(\Delta t)$ curves is achieved. In this recursive feedback method, specifically for $\tilde{\gamma} \approx 1$ and $\tilde{\gamma} \ll 1$, one should remember to compare the theoretical and the simulation $\mathcal{P}(\Delta t)$ curves for 0 $<\Delta t \lesssim 1 / v_{N}$ while obtaining the numerical value of $k_{m}$, since for $\Delta t \sim 2 / v_{N}$, there are other complications that come to play a very significant role. These effects are discussed in Sec. IV C.

[34] In actuality, at the instant step (b) is over, the population of the $\left(k_{m}-1\right)$ th bin has grown to a value bigger than 1 . As a result, the diffusion of an $X$ particle from the $\left(k_{m}-1\right)$ th bin to the $k_{m}$ th bin takes slightly smaller time than the time scale for a new foremost bin creation $1 / v_{N}$. A $1 / v_{N}$ time scale would be applicable if the population of the $\left(k_{m}-1\right)$ th bin is exactly 1 at the instant step (b) is over. We choose to ignore this, and by choosing to do so, we overestimate the value of $t_{0}$ by a small amount.

[35] Of course, in an actual process, $t_{0}$ is determined probabilistically and not in such a sharp manner. However, we should remember that this is only an estimate, and we will show later that it works reasonably well.
[36] For large values of $\tilde{\gamma}$, there can be another kind of event, where the vacant foremost bin event takes place more than once for the same foremost bin. This is more likely for large values of $\tilde{\gamma}$, since the hops from the foremost bin towards the left is much more likely before the number of $X$ particles gets a chance to grow in the foremost bin. In that case, one can simply extend this existing way of estimating $t_{0}$ to $t_{0} \approx 4 / v_{N}$, $t_{0} \approx 6 / v_{N}, \ldots$, etc.

[37] The sequence of random numbers generated by drand48 is random enough for our purposes so long as we use any large enough $\left(\sim 10^{6}\right)$ initial seed. There is nothing special about choosing 123456 , it is as arbitrarily chosen as any other initial seed $\sim 10^{6}$. We have also run the simulations for $N=10^{4}$ and $\tilde{\gamma}=1$ with two other random seeds, namely, $s=10^{6}$ and $s$ $=10^{7}$. For both of these two cases, the average speeds calculated by means of Eq. (5.7) fell within the error bars of Eq. (5.8). Thus, the chance of our results being affected by inherent correlations of the random number generator provided in the standard C library is ruled out.

[38] We have verified that at $k_{b}$, the front shape is still given by the solution of the linear equation, Eq. (4.6).

[39] W.H. Press, B.P. Flannery, S.A. Teukolsky, and W.T. Veterling, Numerical Recipes (Cambridge University Press, Cambridge, 1986). 